COMMUNICATIONS IN

ANALYSIS AND GEOMETRY

Volume 9, Number 1, 61-95, 2001

\title{
Complex hyperbolic manifolds homotopy equivalent to a Riemann surface
}

William M. Goldman, Michael Kapovich and Bernhard Leeb ${ }^{1}$

We construct isometric actions of fundamental groups of closed Riemann surfaces on the complex hyperbolic plane, which realize all possible values of Toledo's invariant $\tau$. For integer values of $\tau$ these actions are discrete embeddings. The quotient complex hyperbolic surfaces are disc bundles over closed Riemann surfaces, whose topological type is described in terms of $\tau$. We relate our geometric construction to arithmetic constructions and discuss integrality properties of $\tau$.

\section{Introduction.}

Let $\Sigma$ denote a closed oriented surface of genus $g \geq 2$ and $\pi=\pi_{1}(\Sigma)$ its fundamental group. Let $G=\mathrm{PU}(n, 1)$ denote the group of biholomorphic isometries of complex hyperbolic $n$-space $\mathbf{H}_{\mathbb{C}}^{n}$. In [15], Domingo Toledo considered the following invariant associated to a representation $\rho: \pi \rightarrow$ $\operatorname{PU}(n, 1):$ Let $\tilde{f}: \tilde{\Sigma} \rightarrow \mathbf{H}_{\mathbb{C}}^{n}$ be a $\rho$-equivariant smooth mapping of the universal covering of $\Sigma$. Then Toledo's invariant $\tau(\rho ; \tilde{f})$ is defined as the (normalized) integral of the pull-back of the Kähler form $\omega$ on $\mathbf{H}_{\mathbb{C}}^{n}$ :

$$
\tau(\rho ; \tilde{f}):=\frac{1}{2 \pi} \int_{\Omega} \tilde{f}^{*} \omega
$$

where $\Omega$ is a fundamental domain for the action of $\pi$ on $\tilde{\Sigma}$. $\tau(\rho ; \tilde{f})$ is independent of $\tilde{f}$ and depends continuously on the representation $\rho$. Toledo

${ }^{1}$ This research was partially supported by National Science Foundation grants, the University of Maryland Institute for Advanced Computer Studies, the General Research Board of the University of Maryland, the Alfred P. Sloan Foundation, the Mathematical Sciences Research Institute, the Sonderforschungsbereich 256 in Bonn and the Volkswagen-Stiftung (Research-in-Pairs program at the Mathematisches Forschungsinstitut Oberwolfach). 
proved that

$$
2-2 g \leq \tau \leq 2 g-2
$$

He showed furthermore that $|\tau|=2 g-2$ holds if and only if $\rho$ is an isomorphism onto a lattice in the stabilizer of a complex geodesic in $\mathbf{H}_{\mathbb{C}}^{n}$. By relating $\tau(\rho)$ to the first Chern class of a certain line bundle we show that it satisfies the integrality property:

$$
\tau \in \frac{2}{n+1} \mathbb{Z}
$$

The function

$$
\tau: \operatorname{Hom}(\pi, \mathrm{PU}(n, 1)) \longrightarrow \frac{2}{n+1} \mathbb{Z}
$$

is locally constant and the Toledo invariant takes one value on each connected component of the representation space. Xia [16] shows that, vice versa, the Toledo invariant distinguishes different connected components. The main results of this paper concern the values realized by Toledo's invariant:

Theorem 1.1. For every genus $g \geq 2$ and every $\tau$ satisfying (1.1) and (1.2) there exists a representation $\rho: \pi \rightarrow \mathrm{PU}(2,1)$ with $\tau(\rho)=\tau$.

When $\tau$ is not an integer, then $\rho$ is not faithful in our examples (but $\rho(\pi)$ is discrete when $\tau= \pm 4 / 3)$. However, when $\tau$ is an even integer, we find discrete embeddings:

Theorem 1.2. For every genus $g \geq 2$ and every even integer $\tau$ satisfying (1.1) there exists a convex-cocompact discrete and faithful representation $\rho: \pi \rightarrow \mathrm{PU}(2,1)$ with $\tau(\rho)=\tau$. Furthermore, the complex hyperbolic surface $M=\mathbf{H}_{\mathbb{C}}^{2} / \rho(\pi)$ is diffeomorphic to the total space of an oriented $\mathbb{R}^{2}$-bundle $\xi$ over $\Sigma$ with the Euler number

$$
e(\xi)=\chi(\Sigma)+|\tau(\rho) / 2|
$$

Generally, for a discrete embedding $\pi \rightarrow \mathrm{PU}(2,1)$ the equality (1.3) will fail [8].

Simple examples of complex hyperbolic surfaces arise from representations preserving totally geodesic planes. There are two kinds of totally geodesic submanifolds $W \subset \mathbf{H}_{\mathbb{C}}^{2}$ of real dimension two: 
- Complex geodesics (copies of $\mathbf{H}_{\mathbb{C}}^{1}$ ) have constant sectional curvature -1 . Their stabilizers in $\mathrm{PU}(2,1)$ are conjugate to $\mathrm{U}(1,1)$.

- Totally real geodesic 2-planes (copies of $\mathbf{H}_{\mathbb{R}}^{2}$ ) have constant sectional curvature $-1 / 4$. Their stabilizers in $\mathrm{PU}(2,1)$ are conjugate to $\mathrm{SO}(2,1)$.

Let $W$ be such a totally geodesic submanifold and $G$ its stabilizer. For a surface group $\pi$, there exist discrete embeddings $\rho: \pi \longrightarrow G$ whose image is a cocompact lattice in $G$. When $W$ is a complex line, then $|\tau(\rho)|=2 g-2$ is maximal; we say that $\rho$ is complex Fuchsian. When $W$ is a totally real plane, then $\tau(\rho)=0$; then we say that $\rho$ is real Fuchsian. These two special cases are building blocks in our constructions.

Quotients of $\mathbf{H}_{\mathbb{C}}^{2}$ by real and complex Fuchsian groups provide two quite different kinds of complex hyperbolic surfaces. For any manifold $M_{1}$ which is the quotient space of the complex Fuchsian group, contains $\Sigma$ as a totally geodesic complex curve. In contrast, the quotients $M_{2}$ of the real Fuchsian groups are Stein manifolds (see Burns and Shnider [2] or Goldman [5],§5.4.7). Moreover, the surfaces $M_{1}$ and $M_{2}$ are not even homeomorphic. They are both diffeomorphic to total spaces of oriented 2-plane bundles over $\Sigma$. The Euler number of the bundle equals $\chi(\Sigma) / 2$ for $M_{1}$ and $\chi(\Sigma)$ for $M_{2}$. These two examples are discussed in detail in $\S \S 2.3-2.5$.

The examples in Theorem 1.2 amalgamate real and complex Fuchsian representations. The representations in Theorem 1.1 amalgamate pairs of holonomy representations of hyperbolic cone structures on closed surfaces.

The present paper is an extended and corrected version of the preprint [6].

Acknowledgements. We thank Domingo Toledo for helpful discussions. Conversations with Igor Belegradek, Jeff Hakim, Steve Kudla, John Millson, Alan Reid, Larry Triplett and Eugene Xia have also been useful. Finally we would like to thank the referee for several important suggestions.

\section{Representations of surface groups in $P U(2,1)$ and their characteristic numbers.}

\subsection{Toledo's invariant.}

We refer to [5] and Toledo's papers [14, 15] for the necessary background for this section. (A general description of characteristic classes associated to representations may also be found in Dupont's paper [3].) Let $G:=$ 
$\mathrm{PU}(n, 1), X:=\mathbf{H}_{\mathbb{C}}^{n}$ and $\pi$ is the fundamental group of a closed Riemann surface $\Sigma$ of genus $g \geq 2$. In this paper we will use the unit ball model for $\mathbf{H}_{\mathbb{C}}^{n}$, whereby

$$
\mathbf{H}_{\mathbb{C}}^{n} \subset \mathbb{C}^{n} \subset \mathbb{P}_{\mathbb{C}}^{n}
$$

For any $\rho \in \operatorname{Hom}(\pi, G)$ we have the associated flat $\mathbf{H}_{\mathbb{C}}^{n}$-bundle $X_{\rho}$ over $\Sigma$ with holonomy $\rho$. Its total space is the quotient of $\tilde{\Sigma} \times X$ by the action of $\pi=\pi_{1}(\Sigma)$ given by

$$
\gamma:(\tilde{m}, x) \mapsto(\gamma \tilde{m}, \rho(\gamma) x)
$$

The Kähler form on $X=\mathbf{H}_{\mathbb{C}}^{n}$ defines a closed 2-form $\omega_{\rho}$ on $X_{\rho}$. (In the above description, $\omega_{\rho}$ satisfies $\Pi^{*} \omega_{\rho}=\Pi_{X}^{*} \omega$ where

$$
\Pi_{X}: \tilde{\Sigma} \times X \longrightarrow X
$$

and

$$
\Pi: \tilde{\Sigma} \times X \longrightarrow X_{\rho}
$$

are the natural projections.) For any section $s: \Sigma \rightarrow X_{\rho}$,

$$
\tau(\rho ; s)=\frac{1}{2 \pi} \int_{\Sigma} s^{*} \omega_{\rho}
$$

is independent of $s$ and is thus an invariant of $\rho$.

Conjugating by an antiholomorphic isometry of $\mathbf{H}_{\mathbb{C}}^{n}$ yields representations with opposite characteristic number. (An example of an antiholomorphic isometry is the complex conjugation $z \mapsto \bar{z}$.) Suppose $\rho \in \operatorname{Hom}(\pi, G)$, and $\iota \in \operatorname{Isom}\left(\mathbf{H}_{\mathbb{C}}^{n}\right)$ is an antiholomorphic isometry. Then $\iota^{*} \omega=-\omega$ and it follows that $\tau\left(\rho^{\iota}\right)=-\tau(\rho)$.

Recall that $\rho$ is called a discrete embedding if its image is a discrete subgroup of $G$ and $\rho$ is injective. In case $\rho$ is a discrete embedding, the quotient $M=\mathbf{H}_{\mathbb{C}}^{n} / \rho(\pi)$ is a complex hyperbolic manifold. A section $s$ of the flat bundle $X_{\rho}$ corresponds to a homotopy equivalence $f: \Sigma \longrightarrow M$ as follows. Lifting everything to the universal covering $\tilde{\Sigma}$, the section $s$ of $X_{\rho}$ defines a section $\tilde{s}: \tilde{\Sigma} \longrightarrow \tilde{\Sigma} \times X$ which is the graph of a $\rho$-equivariant map $\tilde{f}: \tilde{\Sigma} \longrightarrow X$. Equivariance implies that $\tilde{f}$ covers a map $f: \Sigma \longrightarrow M$ which induces the isomorphism

$$
\pi_{1}(\Sigma) \longrightarrow \rho\left(\pi_{1}(\Sigma)\right) \cong \pi_{1}(M) .
$$

Since both $\Sigma$ and $M$ are aspherical, $f$ is a homotopy equivalence. Thus

$$
\tau(\rho)=\frac{1}{2 \pi} \int_{\Sigma} s^{*} \omega_{\rho}=\frac{1}{2 \pi} \int_{\Sigma} f^{*} \omega_{M}
$$




\subsection{Integrality.}

Let $L \rightarrow \mathbf{H}_{\mathbb{C}}^{n}$ be a Hermitian complex line bundle, and let $G$ be a Lie group acting on $L$ by isomorphisms. Let $\Omega$ denote the curvature form of $L$; it is a $G$-invariant 2-form on $\mathbf{H}_{\mathbb{C}}^{n}$.

Given a representation $\rho: \pi \rightarrow G$ and an equivariant smooth map $f:$ $\tilde{\Sigma} \rightarrow \mathbf{H}_{\mathbb{C}}^{n}$, the group $\pi$ acts on the pull-back bundle $f^{*} L \rightarrow \tilde{\Sigma}$, and $f^{*} L$ descends to a Hermitian line bundle $L_{\rho} \rightarrow \Sigma$. The curvature form of $f^{*} L$ is given by $f^{*} \Omega$ and therefore $\frac{1}{2 \pi i} f^{*} \Omega$ descends to a 2 -form $\alpha_{\rho}$ on $\Sigma$ representing the first Chern class $c_{1}\left(L_{\rho}\right)$. Hence

$$
\int_{\Sigma} \alpha_{\rho}=c_{1}\left(L_{\rho}\right) \in \mathbb{Z}
$$

We apply this remark now in two concrete situations. Let $\kappa \rightarrow \mathbf{H}_{\mathbb{C}}^{n}$ be the canonical line bundle and $\theta \rightarrow \mathbf{H}_{\mathbb{C}}^{n}$ be the restriction to $\mathbf{H}_{\mathbb{C}}^{n}$ of the tautological bundle

$$
\mathbb{C}^{n+1} \backslash\{0\} \rightarrow P_{n}(\mathbb{C})
$$

over projective $n$-space. Then $\theta^{n+1} \cong \kappa$.

There are canonical actions of $\mathrm{SU}(n, 1)$ on $\theta$ and of $\mathrm{PU}(n, 1)$ on $\kappa$. Both bundles carry invariant Hermitian metrics which are unique up to scaling. The corresponding curvature forms are given by

$$
\Omega(\theta)=\frac{1}{2 i} \omega
$$

respectively

$$
\Omega(\kappa)=\frac{n+1}{2 i} \omega
$$

where $\omega$ denotes the Kähler form on $\mathbf{H}_{\mathbb{C}}^{n}$.

Given a representation $\rho: \pi \rightarrow \mathrm{PU}(n, 1)$ we consider the line bundle $L_{\rho} \rightarrow \Sigma$ associated to $\kappa \rightarrow \mathbf{H}_{\mathbb{C}}^{n}$. We obtain

$$
c_{1}\left(L_{\rho}\right)=\int_{\Sigma} \alpha_{\rho}=\frac{1}{2 \pi i} \int_{\Sigma} f^{*} \Omega(\kappa)=-\frac{n+1}{4 \pi} \int_{\Sigma} f^{*} \omega=-\frac{n+1}{2} \tau(\rho),
$$

So

$$
\tau(\rho) \in \frac{2}{n+1} \cdot \mathbb{Z}
$$


If $\rho$ lifts to a representation $\tilde{\rho}: \pi \rightarrow \mathrm{SU}(n, 1)$ consider instead the line bundle $L_{\tilde{\rho}} \rightarrow \Sigma$ associated to $\theta \rightarrow \mathbf{H}_{\mathbb{C}}^{n}$, obtaining

$$
c_{1}\left(L_{\tilde{\rho}}\right)=-\frac{1}{2} \tau(\rho)
$$

so

$$
\tau(\rho) \in 2 \cdot \mathbb{Z}
$$

\subsection{Example: Complex lines.}

Basic examples of representations $\pi \rightarrow \mathrm{PU}(n, 1)$ arise from compact complex hyperbolic 1-manifolds embedded in complete complex-hyperbolic surface. We represent complex hyperbolic space $\mathbf{H}_{\mathbb{C}}^{n}$ by the subset of projective space of the indefinite Hermitian vector space $\mathbb{C}^{n, 1}$ of index one and dimension $n+1$ corresponding to lines whose induced Hermitian form is negative definite. Recall that a complex geodesic in $\mathbf{H}_{\mathbb{C}}^{n}$ is a holomorphic totally geodesic complex curve. A complex geodesic has constant curvature -1 , and is equivalent to $C=\mathbf{H}_{\mathbb{C}}^{1}$ embedded in $\mathbf{H}_{\mathbb{C}}^{n}$ as $\{0\} \times \mathbf{H}_{\mathbb{C}}^{1}$. In terms of the unit ball model, in which $\mathbf{H}_{\mathbb{C}}^{n}$ is represented by $\mathbb{B}^{n} \subset \mathbb{C}^{n}$ as

$$
\left[\begin{array}{c}
z_{1} \\
\vdots \\
z_{n}
\end{array}\right] \longmapsto\left[\begin{array}{c}
z_{1} \\
\vdots \\
z_{n} \\
1
\end{array}\right] \in \mathbb{C}^{n, 1}
$$

Under this identification, a model complex geodesic is the image of

$$
\mathbf{H}_{\mathbb{C}}^{1} \longrightarrow\{0\} \times \mathbb{B}^{1} \longrightarrow \mathbb{C}^{n, 1} z \longmapsto(0, \ldots, 0, z) \longleftrightarrow\left[\begin{array}{c}
0 \\
\vdots \\
0 \\
z \\
1
\end{array}\right]
$$

where $z \in \mathbb{C}$ and $|z|<1$. The stabilizer of $C$ is the image of the embedding $\mathrm{U}(n-1) \times \mathrm{U}(1,1) \hookrightarrow \mathrm{PU}(n, 1)$ obtained as the composition of the embedding

$$
\begin{aligned}
\mathrm{U}(n-1) \times \mathrm{U}(1,1) & \hookrightarrow \mathrm{U}(n, 1) \\
(B, A) & \mapsto\left[\begin{array}{cc}
B & 0 \\
0 & A
\end{array}\right]
\end{aligned}
$$


with the projectivization $\mathbb{P}: \mathrm{U}(n, 1) \longrightarrow \mathrm{PU}(n, 1)$. The kernel of the projectivization homomorphism $\mathbb{P}: \mathrm{U}(n, 1) \longrightarrow \mathrm{PU}(n, 1)$ consists of scalar matrices and is the center of $\mathrm{U}(n, 1)$. There are homomorphisms

$$
\mathrm{SU}(1,1) \hookrightarrow \mathrm{SU}(n, 1) \hookrightarrow \mathrm{U}(n, 1) \stackrel{\mathbb{P}}{\longrightarrow} \mathrm{PU}(n, 1)
$$

The homomorphism $\mathrm{SU}(1,1) \rightarrow \mathrm{PU}(n, 1)$ is an embedding since the image of $\mathrm{U}(1,1)$ in $\mathrm{U}(2,1)$ intersects the kernel of $\mathbb{P}$ in the trivial subgroup.

The biholomorphic isometries of $\mathbf{H}_{\mathbb{C}}^{1}$ comprise the group $\mathrm{PU}(1,1)$. The action of $\mathrm{PU}(1,1)$ on $\mathbf{H}_{\mathbb{C}}^{1}$, however, does not quite extend to $\mathbf{H}_{\mathbb{C}}^{n}$. Rather, the group $\mathrm{SU}(1,1)$ acts on $\mathbf{H}_{\mathbb{C}}^{n}$ by the embedding $\mathrm{SU}(1,1) \rightarrow \mathrm{PU}(n, 1)$ above. The restriction homomorphism

$$
\mathrm{SU}(1,1) \rightarrow \operatorname{Isom}\left(\mathbf{H}_{\mathbb{C}}^{1}\right)
$$

defines a double covering of $\mathrm{SU}(1,1)$ onto $\mathrm{PU}(1,1)$. The nontrivial element of its kernel is the reflection in $\mathbf{H}_{\mathbb{C}}^{1}$ given by

$$
\left(z_{1}, \ldots, z_{n-1}, z_{n}\right) \longmapsto\left(-z_{1}, \ldots,-z_{n-1}, z_{n}\right)
$$

which we call the inversion with respect to $\mathbf{H}_{\mathbb{C}}^{1}$.

Although the inversion restricts to the identity on $\mathbf{H}_{\mathbb{C}}^{1}$, it acts nontrivially on $\mathbf{H}_{\mathbb{C}}^{n}$ and on the normal bundle to $\mathbf{H}_{\mathbb{C}}^{1} \subset \mathbf{H}_{\mathbb{C}}^{n}$. This inversion belongs to the maximal compact subgroup $K$ of $\mathrm{SU}(1,1)$ which is represented by diagonal matrices

$$
\left[\begin{array}{ccc}
I_{n-1} & 0 & 0 \\
0 & \zeta & 0 \\
0 & 0 & \zeta^{-1}
\end{array}\right]
$$

where $|\zeta|=1$. It acts on $\mathbf{H}_{\mathbb{C}}^{n}$ by

$$
\left(z_{1}, \ldots, z_{n}\right) \longmapsto\left(\zeta z_{1}, \ldots, \zeta z_{n-1}, \zeta^{2} z_{n}\right) .
$$

Now we specialize to the case $n=2$. Let $\nu^{1}$ denote the unit normal bundle to $\mathbf{H}_{\mathbb{C}}^{1}$ in $\mathbf{H}_{\mathbb{C}}^{2}$ and $U T$ denote the unit tangent bundle of $\mathbf{H}_{\mathbb{C}}^{1}$. Note that $\mathrm{SU}(1,1)$ acts simply-transitively on $\nu^{1}$. Both the normal and tangent bundle of $\mathbf{H}_{\mathbb{C}}^{1}$ have Hermitian connections induced by the Levi-Civita connection on $\mathbf{H}_{\mathbb{C}}^{2}$. These connections restrict to connections on the corresponding unit bundles.

Lemma 2.1. There exists a 2-fold covering $\nu^{1} \rightarrow U T$ which is equivariant with respect to the homomorphism of Lie groups $p: \mathrm{SU}(1,1) \rightarrow \mathrm{PU}(1,1)$. 
Proof. As the group $\mathrm{PU}(1,1)$ acts simply-transitively on $U T$, the 2-1 projection $p: \mathrm{SU}(1,1) \rightarrow \mathrm{PU}(1,1)$ gives us the required 2-fold covering between corresponding circle bundles.

Corollary 2.2. Consider an elliptic isometry $\theta: \mathbf{H}_{\mathbb{C}}^{2} \rightarrow \mathbf{H}_{\mathbb{C}}^{2}$ which lies in the image of the standard embedding $\mathrm{SU}(1,1) \hookrightarrow \mathrm{PU}(2,1)$, assume that the origin $o$ is a fixed point of $\theta$. Then the angle of rotation of $\theta$ in the tangent line $T_{o} \mathbf{H}_{\mathbb{C}}^{1}$ is twice the angle of rotation of $\theta$ in the normal line $\nu_{o} \mathbf{H}_{\mathbb{C}}^{1}$.

Note that the curvature of the pull-back of the connection on $U T$ via $p$ to $\nu^{1}$ is twice the curvature of the connection on UT. Since the induced metric on $T \mathbf{H}_{\mathbb{C}}^{1}$ has sectional curvature -1 , the curvature 2-form of $T \mathbf{H}_{\mathbb{C}}^{1}$ equals $i \cdot d A$ (where $d A$ denotes the area form). Therefore the curvature 2 -form of the normal bundle of $\mathbf{H}_{\mathbb{C}}^{1}$ in $\mathbf{H}_{\mathbb{C}}^{2}$ equals $\frac{i}{2} d A$.

Suppose now that $\Gamma$ is a torsion-free uniform lattice in $\mathrm{SU}(1,1)$,

$$
\Sigma:=\mathbf{H}_{\mathbb{C}}^{1} / \Gamma
$$

(that is, $\Gamma$ is a complex Fuchsian group), and

$$
M:=\mathbf{H}_{\mathbb{C}}^{2} / \Gamma
$$

where $\Gamma$ embeds in $\mathrm{PU}(2,1)$ by the monomorphism $\mathrm{SU}(1,1) \hookrightarrow \mathrm{PU}(2,1)$.

Corollary 2.3. The Euler number of the normal bundle $\nu_{\Sigma}$ of $\Sigma$ in $M$ equals $\frac{1}{2} \chi(\Sigma)$.

The quotient $M=\mathbf{H}_{\mathbb{C}}^{2} / \rho(\Gamma)$ is a complete complex hyperbolic manifold containing a holomorphic totally geodesic submanifold $\mathbf{H}_{\mathbb{C}}^{1} / \rho(\Gamma) \approx \Sigma$. Orthogonal projection onto $\mathbf{H}_{\mathbb{C}}^{1}$ defines a fibration $\Pi_{M}: M \longrightarrow \mathbf{H}_{\mathbb{C}}^{1} / \Gamma$ whose fibers are totally geodesic complex hyperplanes. The fibration $\Pi_{M}$ is isomorphic to the normal bundle $\nu_{\Sigma}$ via the normal exponential mapping. Thus its Euler number equals $\chi(\Sigma) / 2$.

The Kähler form restricts to the area form on the holomorphic submanifold $\Sigma \subset M$, hence Toledo's invariant of the embedding $\Gamma \hookrightarrow \mathrm{PU}(2,1)$ is $-\chi(\Sigma)=2 g-2$.

We will need a relative version of Corollary 2.3.

Definition 2.4. A biholomorphic isometry $h: \mathbf{H}_{\mathbb{C}}^{2} \rightarrow \mathbf{H}_{\mathbb{C}}^{2}$ will be called a transvection if it has an invariant geodesic $\gamma \subset \mathbf{H}_{\mathbb{C}}^{2}$ and $h$ acts trivially on the normal bundle of $\gamma$. (Note that an element is a transvection iff it is conjugate to an element of $\mathrm{SU}(1,1)$ with positive trace.) 
Suppose that $W_{1} \subset \mathbf{H}_{\mathbb{C}}^{2}$ is a totally geodesic subspace of the curvature -1 . Let $\Upsilon \subset \mathrm{PU}(1,1)=\operatorname{Isom}\left(W_{1}\right)$ be a nonabelian discrete finitely generated free purely hyperbolic subgroup, $W_{1} / \Upsilon$ is an open hyperbolic surface. We make the following assumptions:

- The surface $X=W_{1} / \Upsilon$ has even number of ends;

- $\lambda: \Upsilon=\pi_{1}(X) \rightarrow \mathrm{SU}(1,1) \subset \mathrm{PU}(2,1)$ is an embedding such that the image of any peripheral element is a transvection in $\mathbf{H}_{\mathbb{C}}^{2}$.

In the next section we prove a technical lemma which implies that the first condition is necessary and sufficient for the existence of $\alpha$ satisfying the second condition.

Let $\Sigma \subset W_{1} / \Upsilon$ be the convex core, $M:=\mathbf{H}_{\mathbb{C}}^{2} / \lambda(\Upsilon)$ and let $\nu$ and $\nu^{1}$ denote the normal bundle and the unit normal bundle to $\Sigma$ in $M$. The second hypothesis guarantees a parallel section $\alpha$ of the restriction $\left.\nu^{1}\right|_{\partial \Sigma}$. Following Steenrod [12], the relative Euler number $e(\nu, \alpha)$ is defined as the obstruction to extending $\alpha$ to a nonzero section of $\nu$ over all of $\Sigma$.

Proposition 2.5. The relative Euler number $e(\nu, \alpha)$ equals $\frac{1}{2} \chi(\Sigma)$.

Proof. Let $p: \nu(\Sigma) \rightarrow T(\Sigma)$ denote the projection from the normal to the tangent bundle of $\Sigma$ given above. It is clear that the relative Euler number is independent of the choice of parallel section (since all parallel sections are homotopic). Thus we may assume that the projection $p(\alpha)$ is tangent to the boundary of $\Sigma$. Then $e(T(\Sigma), p(\alpha))$ is the Euler characteristic of $\Sigma$ (it follows for instance from taking the double of $\Sigma$ along its boundary and making a symmetric extension of $\alpha$ to $\Sigma$ and its mirror image in the double). On the other hand, since $p$ is a 2 -fold covering we conclude that $e(\nu, \alpha)=\frac{1}{2} \chi(\Sigma)$.

\subsection{Lifting representations from $\mathrm{PU}(1,1)$ to $\mathrm{SU}(1,1)$.}

Gluing manifolds with geometric structures requires that the structures along the common interface agree. In our particular case, we glue along quotients by cyclic groups of hyperbolic elements. We restrict ourselves to the case of complex-hyperbolic plane $\mathbf{H}_{\mathbb{C}}^{2}$.

To construct surfaces $M$ as in Theorem 1.2 we will glue quotients of domains in $\mathbf{H}_{\mathbb{C}}^{2}$ by subgroups $\Gamma_{1}$ stabilizing a complex geodesic $W_{1}$ and 
quotients of domains by subgroups $\Gamma_{2}$ stabilizing a totally real geodesic 2plane $W_{2}$. The interface along which we glue is the quotient of a tube around a (real) geodesic $W_{0}=W_{1} \cap W_{2}$ by a cyclic subgroup. This cyclic subgroup belongs to the intersection $G_{0}:=G_{1} \cap G_{2}$, where $G_{j}$ is the stabilizer of $W_{j}$ in $\mathrm{PU}(2,1)$. In suitable coordinates we may take $W_{1}=\mathbf{H}_{\mathbb{C}}^{1}, W_{2}=\mathbf{H}_{\mathbb{R}}^{2}$ and $W_{0}=\mathbf{H}_{\mathbb{R}}^{1}$. The corresponding stabilizers are $G_{1}=\mathrm{U}(1,1), G_{2}=\mathrm{SO}(2,1)$ and $G_{0}=\mathrm{SO}(1,1)$ respectively.

Figure 1 depicts a "hybrid" surface which is the union of two hyperbolic half-planes. Figure 2 schematically depicts an embedding of the "hybrid" surface into $\mathbf{H}_{\mathbb{C}}^{2}$. The upper half has curvature -1 and represents the Poincare model; the lower half has curvature $-1 / 4$ and represents the KleinBeltrami model. Geometrically this surface is realized in $\mathbf{H}_{\mathbb{C}}^{2}$ as a subset of the union of the totally geodesic subspace $\mathbf{H}_{\mathbb{C}}^{1}$ (the upper half) and $\mathbf{H}_{\mathbb{R}}^{2}$ (the lower half) which meet in $\mathbf{H}_{\mathbb{R}}^{1}$ (the equator). Geodesics orthogonal to $\mathbf{H}_{\mathbb{R}}^{1}$ are drawn in the two halves, as well as the hypercycles parallel to $\mathbf{H}_{\mathbb{R}}^{1}$.

Although $G_{1}$ is connected, neither $G_{2}$ nor $G_{0}$ is connected. In particular any hyperbolic element represented by a matrix in $\mathrm{SO}(2,1)$ with negative trace does not lie in the identity component. A typical such matrix is:

$$
\left[\begin{array}{ccc}
1 & 0 & 0 \\
0 & -\cosh (t) & -\sinh (t) \\
0 & -\sinh (t) & -\cosh (t)
\end{array}\right]
$$

The corresponding isometry of $\mathbf{H}_{\mathbb{C}}^{2}$ is biholomorphic and restricts to an orientation-preserving isometry of $\mathbf{H}_{\mathbb{C}}^{1}$ (since its restriction to $\mathbf{H}_{\mathbb{C}}^{1}$ is holomorphic). However, its restriction to $\mathbf{H}_{\mathbb{R}}^{1}$ reverses orientation: it is a glidereflection, the composition of transvection along the geodesic $\mathbf{H}_{\mathbb{R}}^{1}$ with "inversion" in $\mathbf{H}_{\mathbb{C}}^{1}$. A hyperbolic element of $\mathrm{SU}(1,1)$ is a transvection if and only if it preserves orientation on an invariant real 2-plane; equivalently it lies in a hyperbolic one-parameter subgroup of $\mathrm{SU}(1,1)$.

Proposition 2.6. Let $\Upsilon \subset \mathrm{PU}(1,1)$ be a finitely generated nonabelian purely hyperbolic discrete group of automorphisms of $\mathbf{H}_{\mathbb{C}}^{1}$ such that $\mathbf{H}_{\mathbb{C}}^{1} / \Upsilon$ has an even number of ends. Then there exists a lift $\lambda: \Upsilon \longrightarrow \mathrm{SU}(1,1)$ such that for each $g \in \Upsilon$ corresponding to a simple loop around an end, $\lambda(g)$ is a transvection.

Proof. If $\mathbf{H}_{\mathbb{C}}^{1} / \Upsilon$ is a closed surface then the subgroup $\Upsilon$ lifts isomorphically to $\mathrm{SU}(1,1)$ (see Petersson [11] or [4]); otherwise $\Upsilon$ is a free group. The 


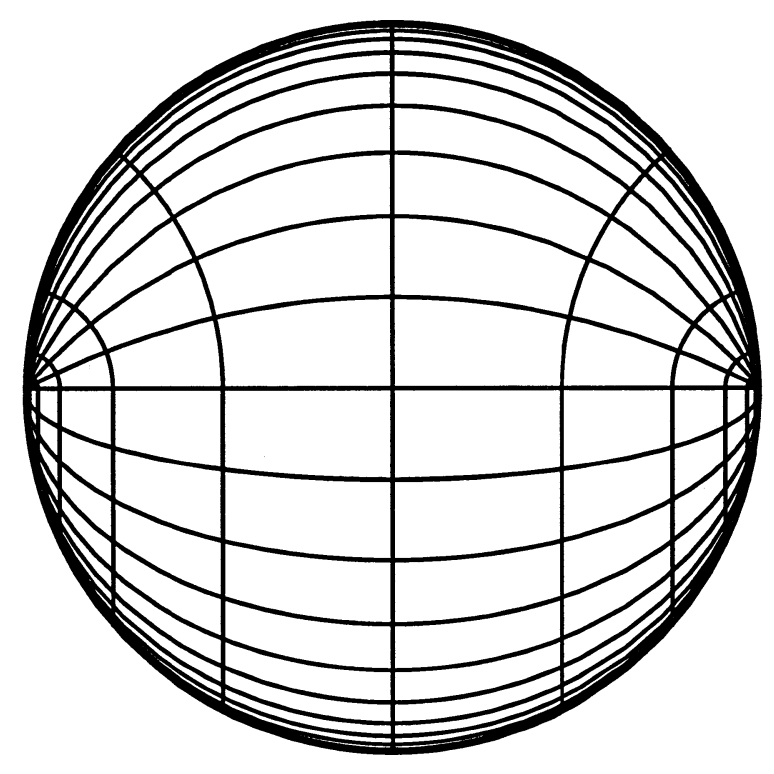

Figure 1: Hybrid surface.

convex core $\Sigma$ of $\mathbf{H}_{\mathbb{C}}^{1} / \Upsilon$ is a compact surface whose boundary consists of closed geodesics $c_{1}, \ldots, c_{m}$ (see [1]).

Choose lifts of the generators of $\Upsilon$ to $\mathrm{SU}(1,1)$ to obtain a homomorphism $\tilde{\lambda}: \Upsilon \longrightarrow \mathrm{SU}(1,1)$ lifting the inclusion $\Upsilon \hookrightarrow \mathrm{SU}(1,1)$. Denote the elements of $\Upsilon$ corresponding to the ends of $\mathbf{H}_{\mathbb{C}}^{1} / \Upsilon$ by $g_{1}, \ldots, g_{m}$. For each $j=1, \ldots, m$ write $\tilde{\lambda}\left(g_{j}\right)=f_{j} \hat{g}_{j}$ where

$$
f_{j}= \pm I \in \mathrm{SU}(1,1)
$$

and $\hat{g}_{j}$ lies in a one-parameter subgroup.

Recall that the normal bundle of $\mathbf{H}_{\mathbb{C}}^{1}$ in $\mathbf{H}_{\mathbb{C}}^{2}$ has a canonical Hermitian connection induced by the Levi-Civita connection on $\mathbf{H}_{\mathbb{C}}^{2}$. This connection 


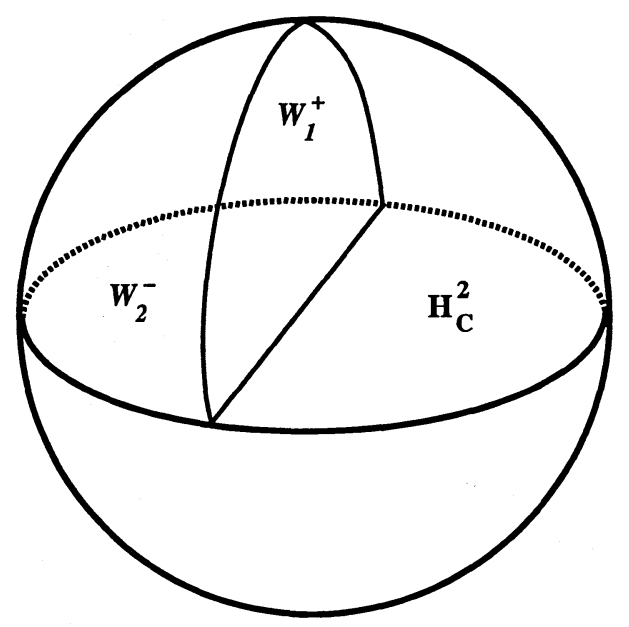

Figure 2: Schematic view of the embedding of hybrid surface into $\mathbf{H}_{\mathbb{C}}^{2}$.

projects to the connection $\nabla$ on the normal bundle $\nu$ of $\Sigma$ in $\mathbf{H}_{\mathbb{C}}^{2} / \Upsilon$.

Choose a basepoint and a loop $l$ which starts at the basepoint and traverses the boundary components $c_{1}, \ldots, c_{m}$. The holonomy of the connection $\nabla$ around $l$ equals the product (of unit complex numbers) $\prod_{j} f_{j}$ of the holonomies around each $c_{j}$. (Compare Kobayashi-Nomizu [10], §II.) By integrating the connection form around $l$ this equals the integral of the curvature form

$$
\exp \left(\int_{\Sigma} \kappa_{\nabla}\right)=\exp \left(\int_{\Sigma} \frac{i}{2} d A\right)=\exp (\pi i \chi(\Sigma))
$$

Since $\chi(\Sigma) \equiv m(\bmod 2)$,

$$
\prod_{j=1}^{m} f_{j}=(-1)^{m}
$$

Since $g_{1}, \ldots, g_{m-1}$ lie in a set $\Delta$ of free generators for $\Upsilon$, there exists a representation $\lambda: \Upsilon \longrightarrow \mathrm{SU}(1,1)$ such that

$$
\lambda\left(g_{j}\right)=f_{j} \tilde{\lambda}\left(g_{j}\right)
$$

for $j<m$ and

$$
\lambda(g)=\tilde{\lambda}(g)
$$


for $g \in \Delta-\left\{\gamma_{1}, \ldots, \gamma_{m}\right\}$. so that $\lambda\left(g_{j}\right)$ are transvections all for $j<m$. Apply (2.1) when $m$ is even to conclude that $\lambda\left(g_{m}\right)$ is also a transvection. This concludes the proof of the proposition.

The referee has suggested the following alternative point of view on the ideas in Proposition 2.6. For a Fuchsian representation into PU $(1,1)$ whose quotient $\Sigma$ is a triply-perforated sphere (a "pair of pants"), the three generators corresponding to the boundary lift to elements of $\mathrm{SU}(1,1)$ with negative trace, as can be deduced from the law of cosines for hyperbolic right-angled hexagons (see, for example, Theorem 7.19.2 of Beardon [1]). By changing the lifting, one can assume that two of the generators have positive trace and the other generator has negative trace.

If $\Sigma$ is a compact hyperbolic surface with totally geodesic boundary, then $\Sigma$ decomposes into pants. Furthermore the number of pants in the decomposition equals $-\chi(\Sigma)$ which has the same parity as the number $m$ of components of $\partial \Sigma$. Since the loops corresponding to $m-1$ components of $\partial \Sigma$ can be included in the set of free generators of $H_{1}(\Sigma, \mathbb{Z})$, we can lift the generators of the fundamental groups of all but one of the boundary components $C_{1}, \ldots, C_{m-1}$ to have positive trace. We show that the the sign of the trace of $\lambda\left(C_{m}\right)$ will be $(-1)^{m}=(-1)^{\chi(\Sigma)}$, by induction on $-\chi(\Sigma)$. Decompose $\Sigma$ into pants, and choose one pants $P$ in the decomposition which intersects $\partial \Sigma$. Apply the induction hypothesis to $\Sigma^{\prime}=\Sigma-P$, whose Euler characteristic equals $1+\chi(\Sigma)$. Now $\Sigma^{\prime}$ has either one less or one more boundary component than $\Sigma$ (depending on how many components of $\partial P$ meet $\partial \Sigma$ ). Suppose first that $\Sigma^{\prime}$ has one more boundary component than $\Sigma$. By induction we assume that one component of $\partial \Sigma^{\prime} \cap \partial P$ lifts to an element of positive trace, the other component of $\partial \Sigma^{\prime} \cap P$ lifts to an element whose trace is $(-1)^{m+1}$ and the components of $\partial \Sigma-P$ all lift to elements of positive trace. It follows from the above paragraph that when $P$ is attached to $\Sigma^{\prime}$, the new boundary component of $P$ will lift to an element of trace $(-1)^{m}$, retaining the lifts of all other boundary components. Similarly, if $\Sigma^{\prime}$ has one less boundary component, then by induction we suppose that all the components of $\partial \Sigma^{\prime}$ have been lifted to elements of positive trace and that $C=\partial \Sigma^{\prime} \cap \partial P$ has been lifted to an element of sign $(-1)^{m+1}$. By the above description of the pants, we can attach $P$ and find a lift of the representation of $\pi_{1}(\Sigma)$ such that one boundary component of $\partial \Sigma \cap \partial P$ has positive trace, the other one has trace of sign $(-1)^{m}$ and all other lifts of components of $\partial \Sigma$ are retained. 


\subsection{Example: Totally real geodesic subspaces.}

At the opposite extreme from holomorphic totally geodesic submanifolds are totally real totally geodesic submanifolds. Every such submanifold is equivalent by an automorphism to real hyperbolic space $\mathbf{H}_{\mathbb{R}}^{2} \subset \mathbf{H}_{\mathbb{C}}^{2}$ - the subset consisting of points with real coordinates. This subspace is the fixedpoint-set of the anti-holomorphic isometric involution

$$
\left(z_{1}, z_{2}\right) \longmapsto\left(\bar{z}_{1}, \bar{z}_{2}\right)
$$

The induced Riemannian metric on $\mathbf{H}_{\mathbb{R}}^{2}$ has constant negative curvature $-1 / 4$. The stabilizer of $\mathbf{H}_{\mathbb{R}}^{2}$ is the image of the embedding $\mathrm{SO}(2,1) \hookrightarrow$ $\mathrm{PU}(2,1)$ obtained by composing the embedding $\mathrm{SO}(2,1) \hookrightarrow \mathrm{U}(2,1)$ with projectivization.

The fibers of the real orthogonal projection $\Pi_{\mathbb{R}}: \mathbf{H}_{\mathbb{C}}^{2} \longrightarrow \mathbf{H}_{\mathbb{R}}^{2}$ are totally real geodesic subspaces which are orthogonal to $\mathbf{H}_{\mathbb{R}}^{2}$. (Compare $\S 3.3 .6$ of Goldman [5].) Since $\mathbf{H}_{\mathbb{R}}^{2}$ is totally real, the almost complex structure defines an $\mathrm{SO}(2,1)$-equivariant isomorphism between its tangent bundle and its normal bundle.

Let $\Gamma \subset \mathrm{SO}(2,1)^{0}$ be a discrete torsion-free subgroup such that the convex core $\Sigma$ of $\mathbf{H}_{\mathbb{R}}^{2} / \Gamma$ is compact. The composition

$$
\rho: \pi_{1}(\Sigma) \cong \Gamma \hookrightarrow \mathrm{SO}(2,1) \subset \mathrm{U}(2,1) \rightarrow \mathrm{PU}(2,1)
$$

is a discrete embedding. The quotient $M=\mathbf{H}_{\mathbb{C}}^{2} / \rho(\Gamma)$ is a complete complex hyperbolic surface containing a totally real totally geodesic submanifold

$$
\mathbf{H}_{\mathbb{R}}^{2} / \rho(\Gamma) \cong \Sigma
$$

The corresponding embedding

$$
\Sigma \hookrightarrow \mathbf{H}_{\mathbb{R}}^{2} / \Gamma \subset M
$$

induces the homomorphism $\rho: \pi_{1}(\Sigma) \cong \Gamma$ of fundamental groups. From now on identify $\Sigma$ with its image under this embedding.

Suppose $\Sigma$ has no boundary. The above discussion implies the fibration $M \rightarrow \Sigma$ induced by the orthogonal projection $\mathbf{H}_{\mathbb{C}}^{2} \longrightarrow \mathbf{H}_{\mathbb{R}}^{2}$ is diffeomorphic as a fiber bundle to the tangent bundle of $\Sigma$. Hence this fibration has Euler number

$$
e(T \Sigma)=\chi(\Sigma)=2-2 g
$$

We will also need a relative version of this statement in the case $\partial \Sigma \neq \emptyset$. Let $\nu(\Sigma)$ be the normal bundle of $\Sigma$ in $M, \alpha$ is a unit normal parallel vector-field along $\partial \Sigma$ in $M$. 
Proposition 2.7. The relative Euler number $e(\nu(\Sigma), \alpha)$ equals the Euler characteristic $\chi(\Sigma)$.

Proof. We repeat the argument from the proof of Proposition 2.3, replacing the 2-fold covering by the isomorphism $J$ between the normal and tangent bundle. This isomorphism will map $\alpha$ to a parallel section $J(\alpha)$ along $\partial \Sigma$. Hence

$$
e(\nu(\Sigma), \alpha)=e(T(\Sigma), J(\alpha))=\chi(\Sigma)
$$

Since $\Sigma$ is embedded as a totally real submanifold, $\left.\omega\right|_{\Sigma}=0$. Thus Toledo's integrand is identically zero so $\tau(\rho)=0$.

\section{Geometric preliminaries.}

\subsection{Orthogonal projections in Hadamard manifolds.}

Let $W$ be a simply-connected complete manifold with sectional curvature $K_{W} \leq-1 / 4$. Let $W_{1}$ and $W_{2}$ be totally geodesic submanifolds of $W$ with non-empty intersection $W_{0}$. Then $W_{0}$ is a totally geodesic submanifold as well. The angle of intersection of $W_{1}$ and $W_{2}$ at $x \in W_{0}$ is defined to be:

$$
\begin{aligned}
\angle_{x}\left(W_{1}, W_{2}\right) & :=\min \left\{\angle\left(v_{1}, v_{2}\right) \mid v_{1} \in T_{x} W_{1}, v_{2} \in T_{x} W_{2}, v_{1} \perp W_{0}, v_{2} \perp W_{0}\right\} \\
& \in[0, \pi / 2]
\end{aligned}
$$

Since the $W_{j}$ are totally geodesic, parallel translation along $W_{0}$ preserves tangency to $W_{j}$. Therefore the angle $L_{x}\left(W_{1}, W_{2}\right)$ is independent of the point $x \in W_{0}$ and we simply write $\angle\left(W_{1}, W_{2}\right)$ for $\angle_{x}\left(W_{1}, W_{2}\right)$.

Denote by $\Pi_{j}: W \rightarrow W_{j}$ the orthogonal projection onto $W_{j}(j=0,1,2)$. For a subset $S \subset X$ in a metric space $X$ let $\operatorname{Nbd}_{r}(S)$ denote the closed $r$ neighborhood of $S$.

Proposition 3.1. Let $\epsilon(\alpha):=2 \cdot \cosh ^{-1}(\csc (\alpha / 2))$ where $\alpha:=\angle\left(W_{1}, W_{2}\right)$. Then for all $\epsilon>\epsilon(\alpha)$ we have:

$$
W=\Pi_{1}^{-1}\left(\operatorname{Nbd}_{\epsilon}\left(W_{0}\right)\right) \cup \Pi_{2}^{-1}\left(\operatorname{Nbd}_{\epsilon}\left(W_{0}\right)\right)
$$

and the distance between boundary components of

$$
\Pi_{1}^{-1}\left(\operatorname{Nbd}_{\epsilon}\left(W_{0}\right)\right) \cap \Pi_{2}^{-1}\left(\operatorname{Nbd}_{\epsilon}\left(W_{0}\right)\right)
$$

is positive. 
Proof. Pick a point $p \in W$. Consider the projections $p_{j}=\Pi_{j}(p)$ to $W_{j}$ $(j=0,1,2)$ and let $q=\Pi_{0}\left(p_{1}\right) \in W_{0}$. It suffices to show that $d\left(p_{1}, q\right) \leq \epsilon$ or $d\left(p_{2}, q\right) \leq \epsilon$.

Denote by $u_{j} \in T_{q}\left(W_{j}\right)$ the unit tangent vector at $q$ pointing towards $p_{j}$. Then $u_{1} \perp W_{0}$. Let $u_{2}^{\prime} \in T_{q} W_{2}$ be the component of $u_{2}$ orthogonal to $W_{0}$. Then

$$
\left|\left\langle u_{1}, u_{2}\right\rangle\right|=\left|\left\langle u_{1}, u_{2}^{\prime}\right\rangle\right| \leq\left|u_{2}^{\prime}\right| \cos (\alpha)
$$

and we get $\alpha \leq \angle\left(p_{1} q p_{2}\right) \leq \angle\left(p_{1} q p\right)+\angle\left(p q p_{2}\right)$. Hence, we assume without loss of generality that $q \in W_{0}$ satisfies

$$
\angle\left(p_{1} q p\right) \geq \frac{\alpha}{2}
$$

Let $\mathbf{H}_{\mathbb{R}}^{2}$ be the hyperbolic plane with sectional curvature $-1 / 4$. Consider a. triangle $\triangle\left(\tilde{p}_{1} \tilde{q} \tilde{p}\right)$ in $\mathbf{H}_{\mathbb{R}}^{2}$ having the same side lengths as $\triangle\left(p_{1} q p\right)$. (Compare Figure 3.) Since $W$ has sectional curvature bounded above by $-1 / 4$, angle comparison with $\mathbf{H}_{\mathbb{R}}^{2}$ implies (see [9])

$$
\angle\left(\tilde{p}_{1} \tilde{q} \tilde{p}\right) \geq \angle\left(p_{1} q p\right) \geq \frac{\alpha}{2} \quad \text { and } \quad \angle\left(\tilde{q} \tilde{p}_{1} \tilde{p}\right) \geq \angle\left(q p_{1} p\right)=\frac{\pi}{2} .
$$

(by (3.2)). Hence

$$
d\left(p_{1}, q\right)=d\left(\tilde{p}_{1}, \tilde{q}\right) \leq \epsilon(\alpha)
$$

where $\epsilon=\epsilon(\alpha)$ is the length of the finite side of the right ideal triangle in $\mathbf{H}_{\mathbb{R}}^{2}$ with angle $\alpha / 2$. For such a triangle (see, for example Beardon [1], Theorem 7.9.1)

$$
\cosh \left(\frac{\epsilon}{2}\right)=\csc \left(\frac{\alpha}{2}\right)
$$

whence the first assertion of the proposition follows. In particular for each $\epsilon>\epsilon(\alpha)$ we have:

$$
\partial \Pi_{1}^{-1}\left(\operatorname{Nbd}_{\epsilon}\left(W_{0}\right)\right) \cap \partial \Pi_{2}^{-1}\left(\operatorname{Nbd}_{\epsilon}\left(W_{0}\right)\right)=\emptyset
$$

To show that the distance between these hypersurfaces is positive choose $\delta$ such that $\epsilon(\alpha)<\delta<\epsilon$. Recall that the nearest-point projection in Hadamard spaces does not increase the distance. Hence for $j=1,2$ the set

$$
U_{j, \delta}:=\Pi_{j}^{-1}\left(W_{0}-\operatorname{Nbd}_{\delta}\left(W_{0}\right)\right)
$$

contains the $(\epsilon-\delta)$-neighborhood of the hypersurface $\partial \Pi_{j}^{-1}\left(\operatorname{Nbd}_{\epsilon}\left(W_{0}\right)\right)$ and as we already proved $U_{1, \delta} \cap U_{2, \delta}=\emptyset$. The second assertion of the proposition follows. 


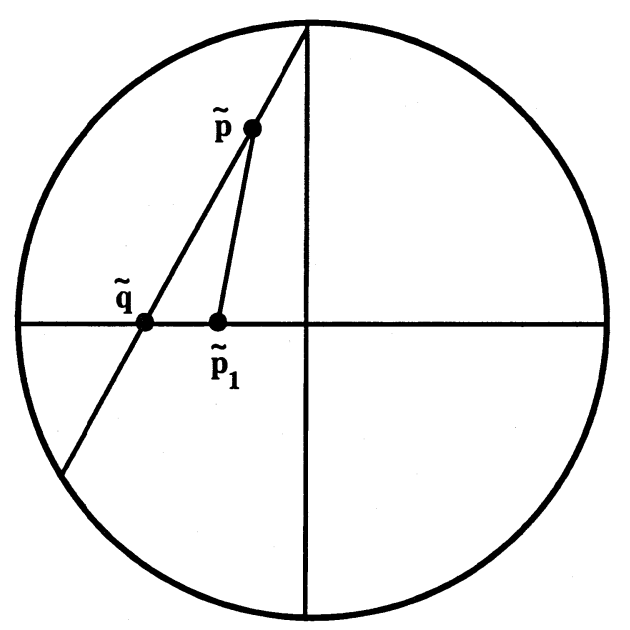

Figure 3: The comparison triangle

\subsection{A model for the neck.}

In this section we discuss the topology of special neighborhoods of closed real geodesics in complex-hyperbolic surfaces; these neighborhoods are called necks.

First we describe infinitesimal data associated with each neck. Let $h$ be a transvection in $\mathbf{H}_{\mathbb{C}}^{2}$ along a real geodesic $W_{0}$. Consider the quotient $M:=\mathbf{H}_{\mathbb{C}}^{2} /\langle h\rangle:$ it contains the closed geodesic $\gamma:=W_{0} /\langle h\rangle$, let $\ell$ be the length of $\gamma$. The choice of the generator $h$ of the cyclic group $\langle h\rangle$ corresponds to the choice of an orientation on $\gamma$. Hence, pick a point $x \in \gamma$ and a unit tangent vector $v_{1} \in T_{x}(\gamma) \subset T_{x}(M)$ pointing in the positive direction. Then choose a unit tangent vector $v_{2} \in T_{x}(M)$ which is Hermitian orthogonal to $v_{1}$. The pair $\left(v_{1}, v_{2}\right)$ can be canonically completed to an orthonormal basis of $T_{x}(M)$ over $\mathbb{R}$ : let

$$
w_{1}:=\sqrt{-1} v_{1}, w_{2}:=\sqrt{-1} v_{2} .
$$

We call $\left(v_{1}, v_{2}\right)$ a normal frame at $x$. Note that our assumptions on $\gamma$ imply that the normal frame canonically extends to a smooth parallel frame field along $\gamma$.

Such a normal frame and the number $\epsilon>0$ uniquely determine a neck $N(\epsilon, \ell)$ in the manifold $M$ around $\gamma$ as follows:

- Any lift $\tilde{w}_{1}$ of $w_{1}$ to $\left.T\left(\mathbf{H}_{\mathbb{C}}^{2}\right)\right|_{W_{0}}$ is tangent to the unique complex geodesic $W_{1}$ in $\mathbf{H}_{\mathbb{C}}^{2}$ containing $W_{0}$. 
- Any lift $\tilde{v}_{2}$ of $v_{2}$ to $\left.T\left(\mathbf{H}_{\mathbb{C}}^{2}\right)\right|_{W_{0}}$ is tangent to a totally geodesic realhyperbolic plane $W_{2}$ in $\mathbf{H}_{\mathbb{C}}^{2}$ containing $W_{0}$.

- The vectors $\tilde{w}_{1}, \tilde{v}_{2}$ determine half-planes in

$$
W_{1}^{+} \subset W_{1}, W_{2}^{+} \subset W_{2}
$$

bounded by $W_{0}$. (The vectors point outside these half-planes).

We denote by $X_{j}$ the union of the open half-plane $W_{j}^{+}$and the closed $\epsilon$ neighborhood of $W_{0}$ in $W_{j}$. Then $S_{j}:=X_{j}-W_{j}^{+}$is a strip bounded by $W_{0}$ and a hypercycle $h_{j}$ equidistant from $W_{0}$. Denote by $Y_{j}$ the inverse image of $X_{j}$ under the orthogonal projection $\Pi_{j}: \mathbf{H}_{\mathbb{C}}^{2} \rightarrow W_{j}$. The next lemma follows directly from Proposition 3.1.

Lemma 3.2. Let

$$
\epsilon>\epsilon_{0}=\epsilon(\pi / 2)=2 \ln (1+\sqrt{2})=\ln (3+2 \sqrt{2}) .
$$

Then $Y_{1} \cup Y_{2}=W$ and moreover the distance between the boundary components of $Y_{1} \cap Y_{2}$ is positive.

Let $\epsilon_{1}:=\ln (6)>\epsilon_{0}$ and suppose in the following that $\epsilon>\epsilon_{1}$. The 1-parameter group $\mathrm{O}(1,1)^{0}$ of transvections along the geodesic $W_{0}$ acts on the intersection $Y(\epsilon)=Y=Y_{1} \cap Y_{2}$. We call the intersection $Y$ a tube.

We describe the topology of the tube $Y$ in an $\mathrm{O}(1,1)^{0}$-invariant way. The group $\mathrm{O}(1,1)^{0}$ acts freely on the smooth manifold with boundary $\mathbf{H}_{\mathbb{C}}^{2} \cup$ $\partial_{\infty} \mathbf{H}_{\mathbb{C}}^{2}-\partial_{\infty} W_{0}$ which can be thought of as the total space of an $\mathbb{R}$-principal bundle $E$ whose base is the closed 3-ball $B^{3}$. Let $\bar{Y}$ be the closure of $Y$ in $\mathbf{H}_{\mathbb{C}}^{2} \cup \partial_{\infty} \mathbf{H}_{\mathbb{C}}^{2}-\partial_{\infty} W_{0}$. It is invariant under the $\mathrm{O}(1,1)^{0}$-action. The frontier $\partial Y$ of $Y$ in $\mathbf{H}_{\mathbb{C}}^{2} \cup \partial_{\infty} \mathbf{H}_{\mathbb{C}}^{2}-\partial_{\infty} W_{0}$ projects to a union of two disjoint smoothly properly embedded 2-discs in $B^{3}$. Hence, by the smooth Schönflies Theorem in dimension three, the projection of $\bar{Y}$ is diffeomorphic to $D^{2} \times$ $[1,2]$. Since $E$ is a trivial bundle it has a smooth trivialization. It can be chosen to extend the canonical trivializations of $\partial Y$ whose horizontal sections are totally geodesic 2-planes. This allows us to extend the fibration of $\partial Y$ by totally geodesic 2 -planes in an $\mathrm{O}(1,1)^{0}$-invariant manner to a smooth fibration of $\bar{Y}$ by closed 2-discs. As base for this bundle we can use the strip $S_{1} \cup S_{2}$. The above discussion shows:

Proposition 3.3. The quotient $\bar{Y} /\langle h\rangle$ is diffeomorphic to the total space of a trivial disk bundle over the annulus $A$. The restriction of this fibration to 
the two components of $\partial Y /\langle\gamma\rangle$ corresponds to the restriction of the nearestpoint projections $\Pi_{j}$ to $\Pi^{-1}\left(h_{j}\right), j=1,2$.

We call the quotient $N(\epsilon, \ell)=N:=Y /\langle h\rangle$ a model neck. Its isometry type depends on two parameters: $\epsilon$ (the width) and $\ell$ (the length).

\subsection{Collars of geodesics.}

In this section we determine $\ell=\ell(D)>0$ such that a closed geodesic of length $\ell$ in a hyperbolic surface of curvature $-k^{-2}$ is contained in an regular tubular neighborhood (a "collar") of width at least $D$ and distinct neighborhoods are disjoint. This will enable us to find a neck along which to glue the two complex hyperbolic surfaces with holomorphic and totally real spines respectively. For simplicity we consider only surfaces where all boundary geodesics have the same length.

Let $W_{k}$ be a complete simply connected Riemannian 2-manifold of constant curvature $-k^{-2}$ and $\Gamma_{k}$ a purely hyperbolic discrete group of isometries. Suppose that $f, g$ are nontrivial elements of $\Gamma_{k}$ which are transvections along geodesics $A_{f}, A_{g}$ in $W_{k}$. The geodesics $A_{f}, A_{g}$ are called the axes of $f, g$.We assume that:

- The geodesics $A_{f}, A_{g}$ are disjoint.

- The geodesics $A_{f} /\langle f\rangle, A_{g} /\langle g\rangle$ have the same length $\ell$.

\section{Lemma 3.4.}

$$
\sinh \left(\frac{\ell}{2 k}\right) \sinh \left(\frac{d\left(A_{f}, A_{g}\right)}{2 k}\right) \geq 1 / 2
$$

Proof. This assertion quantifies the Margulis Lemma for the hyperbolic plane and slightly modifies Corollary 11.6.10 of Beardon [1]. Beardon considers the case of curvature -1 but the formulas remain valid for constant curvature $K<0$ if the lengths are multiplied by $\sqrt{-K}$.

Let $S$ be a complete hyperbolic surface and $\gamma$ a simple closed geodesic in $S$. Let $S_{\gamma} \rightarrow S$ denote the locally isometric covering of $S$ corresponding to the subgroup of $\pi_{1}(S)$ generated by $\gamma$. Let $\hat{\gamma}$ denote the simple closed geodesic in $S_{\gamma}$ covering $\gamma$. We say that the $\epsilon$-neighborhood $\operatorname{Nbd}_{\epsilon}(\gamma)$ of $\gamma$ in $S$ is injective if the projection

$$
\operatorname{Nbd}_{\epsilon}(\hat{\gamma}) \rightarrow \operatorname{Nbd}_{\epsilon}(\gamma)
$$


is a diffeomorphism. Equivalently, $\alpha$ is contained in $\gamma$ for every geodesic arc $\alpha$ in $S$ with both end-points on $\gamma$ and $\ell(\alpha) \leq 2 \epsilon$. Suppose that $\Gamma_{k}$ is a finitely-generated group as in Lemma $3.4, \Sigma_{k}$ is the compact convex core of the surface $S_{k}=W_{k} / \Gamma_{k}$. We assume that all boundary geodesics of $\Sigma_{k}$ have the same length $\ell$. Lemma 3.4 implies:

Lemma 3.5. Let $\epsilon>0$ and suppose that $\ell$ satisfies

$$
\sinh \left(\frac{\ell}{2 k}\right) \sinh \left(\frac{\epsilon}{k}\right) \leq \frac{1}{2},
$$

Then the $\epsilon$-neighborhood of each boundary geodesic of $\Sigma_{k}$ is injective and $\epsilon-n e i g h b o r h o o d s$ of distinct geodesics are disjoint.

Remark 3.6. In the case of equality in (3.4)

$$
\sinh \left(\frac{\ell_{k}}{2 k}\right) \sinh \left(\frac{\epsilon}{k}\right)=\frac{1}{2}
$$

then $\ell_{1}<\ell_{2}$. If $\epsilon=\epsilon_{1}=\ln (6)$ then $\sinh \left(\ell_{1} / 2\right)=6 / 35$.

\subsection{Neck neighborhoods.}

Now we combine the results of the previous three sections to construct neck neighborhoods around certain geodesics in complex-hyperbolic surfaces.

Definition 3.7. Let $M$ be a complex-hyperbolic surface and $\gamma \subset M$ be a simple closed geodesic of length $\ell$. We say that $\gamma$ admits an $(\epsilon, l)$-neck neighborhood $\operatorname{Neck}_{\epsilon}(\gamma)$ of width $\epsilon$ in $M$ if it has a neighborhood in $M$ which is isometric to the model neck $N(\epsilon, \ell)$.

Suppose that $W_{k} \subset W=\mathbf{H}_{\mathbb{C}}^{2}$ is a totally geodesic plane of the curvature $-k^{-2}$ and $\Gamma_{k}$ is a finitely-generated purely loxodromic discrete subgroup of $\mathrm{PU}(2,1)$ stabilizing $W_{k}$. We assume that $S_{k}=W_{k} / \Gamma_{k}$ is not compact and $\Sigma_{k} \subset S_{k}$ is the compact convex core so that the length of each boundary geodesic equals the same number $\ell$. We also assome that each element of $\Gamma_{k}$ representing a boundary geodesic $\gamma$ of $\Sigma_{k}$ is a transvection.

To construct the infinitesimal data for the neck around each boundary geodesic $\gamma$ of $\Sigma_{k}$ choose a normal frame at $\gamma \subset \mathbf{H}_{\mathbb{C}}^{2} / \Gamma_{k}$ (see subsection 3.2):

- Pick a base point $x$ at $\gamma$. 
- The vector $v_{1}$ tangent to $\gamma$ at $x$ is determined by the choice of orientation on $\gamma$, we let $w_{1}:=\sqrt{-1} v_{1}$. If $k=1$ then the vector $w_{1}$ is tangent to $\Sigma_{1}$ and is directed inwards $\Sigma_{1}$.

- If $k=2$ then the second vector $v_{2}$ is chosen tangent to $\Sigma_{2}$ and directed inwards $\Sigma_{2}$.

Lemma 3.8. Suppose that $\ell<\ell_{1}$, where $\ell_{1}$ is given by

$$
\sinh \left(\ell_{1} / 2\right)=6 / 35
$$

Then for $\epsilon=\epsilon_{1}=\ln (6)$ we have:

- Each boundary geodesic $\gamma \subset \Sigma_{k} \subset \mathbf{H}_{\mathbb{C}}^{2} / \Gamma_{k}$ is contained in a neck neighborhood $\operatorname{Neck}_{\epsilon}(\gamma)$ with the normal frame as above.

- If $\gamma, \beta \subset \partial \Sigma_{k}$ are distinct boundary geodesics then $\operatorname{Neck}_{\epsilon}(\gamma) \cap$ $\operatorname{Neck}_{\epsilon}(\beta)=\emptyset$.

Proof. The condition $\epsilon=\epsilon_{1}>\epsilon_{0}=2 \ln (1+\sqrt{2})$ implies the existence of a model neck of the width $\epsilon$ (Lemma 3.2). Let $\gamma$ be a boundary geodesic of $\Sigma_{k}$ and let

$$
M_{\gamma} \rightarrow M=\mathbf{H}_{\mathbb{C}}^{2} / \Gamma_{k}, \quad \Sigma_{k, \gamma} \rightarrow \Sigma_{k}
$$

denote the coverings defined by the subgroups

$$
\pi_{1}(\gamma) \subset \pi_{1}(M), \quad \pi_{1}(\gamma) \subset \pi_{1}\left(\Sigma_{k}\right) .
$$

Define $\hat{\gamma}$ as the simple closed geodesic on $\Sigma_{k, \gamma} \subset M_{\gamma}$ covering $\gamma$. Lift of the normal frame along $\gamma$ from $M$ to a normal frame along $\hat{\gamma}$ in $M_{\gamma}$ determines an neck neighborhood $\operatorname{Neck}_{\epsilon}(\hat{\gamma}) \subset M_{\gamma}$. We first show that the restriction of the projection $M_{\gamma} \rightarrow M$ to $\operatorname{Neck}_{\epsilon}(\hat{\gamma})$ is injective. Let $\tilde{P}: M_{\gamma} \rightarrow \tilde{\Sigma}_{k}$ denote the nearest-point projection to the lift $\tilde{\Sigma}_{k}$ of $\Sigma_{k}$ into $M_{\gamma}$. Then $\operatorname{Neck}_{\epsilon}(\hat{\gamma})$ is contained in the inverse image $\tilde{P}^{-1}\left(\operatorname{Nbd}_{\epsilon}(\hat{\gamma})\right)$ where the $\epsilon$-neighborhood is taken within the surface $\tilde{\Sigma}_{k}$. Recall that by Lemma 3.5 and Remark 3.6 the geodesic $\gamma$ in $\Sigma_{k}$ has an injective $\epsilon$-neighborhood. Thus the restriction of the covering $\Sigma_{k, \gamma} \rightarrow \Sigma_{k}$ to $\operatorname{Nbd}_{\epsilon}(\hat{\gamma})$ is injective and the restriction of the covering $M_{\gamma} \rightarrow M$ to $\tilde{P}^{-1}\left(\operatorname{Nbd}_{\epsilon}(\hat{\gamma})\right)$ is injective as well. This implies that we have a neck neighborhood $\operatorname{Neck}_{\epsilon}(\gamma)$ of the geodesic $\gamma$ in $M$. Finally 
we show that neck neighborhoods of distinct geodesics are disjoint. Let $P: M=\mathbf{H}_{\mathbb{C}}^{2} / \Gamma_{k} \rightarrow \Sigma_{k}$ denote the orthogonal projection. Then again

$$
\operatorname{Neck}_{\epsilon}(\gamma) \subset P^{-1}\left(\operatorname{Nbd}_{\epsilon}(\gamma)\right)
$$

for every boundary geodesic $\gamma \subset \Sigma_{k}$. Let $\beta, \gamma \subset \partial \Sigma_{k}$ are distinct boundary components. Then their $\epsilon$-neighborhoods $\operatorname{Nbd}_{\epsilon}(\beta), \operatorname{Nbd}_{\epsilon}(\gamma)$ in $\Sigma_{k}$ are disjoint by Lemma 3.5. Hence and $\operatorname{Neck}_{\epsilon}(\gamma) \cap \operatorname{Neck}_{\epsilon}(\beta)=\emptyset$ as desired.

\section{Proof of the Main Theorem.}

We begin with an oriented closed surface $\Sigma$ with a piecewise hyperbolic structure of the following kind. There is a collection $B$ of disjoint simple closed geodesics on $\Sigma$ such that on each component of $\Sigma-B$ the metric either has constant curvature -1 or constant curvature $-1 / 4$. Let $\Sigma_{k}(k=1,2)$ denote the subsurface of $\Sigma-B$ where the curvature equals $-k^{-2}$. The surfaces $\Sigma_{k}$ could be disconnected. We assume that each loop $\beta \in B$ is adjacent to both $\Sigma_{1}, \Sigma_{2}$. Thus we orient $B$ so that the surface $\Sigma_{1}$ lies to the left from $B$. Our construction involves two assumptions:

- The boundary of each component of $\Sigma_{1}$ has an even number of components.

- All components of $B$ have the same length $\ell<\ell_{1}=\sinh ^{-1}(6 / 35)$.

we introduced that in the previous section

Theorem 4.1. There exists a complete complex hyperbolic surface $M$ together with a piecewise totally geodesic isometric embedding $f: \Sigma \rightarrow M$ such that

- $f$ is a homotopy equivalence.

- The Toledo invariant of $M$ equals $-\chi\left(\Sigma_{1}\right)$.

- $M$ is diffeomorphic to the total space of an oriented disc bundle which has the Euler number $\chi\left(\Sigma_{1}\right) / 2+\chi\left(\Sigma_{2}\right)$.

Outline of proof. We start with an outline of the proof. We embed $\Sigma_{2}$ (respectively $\Sigma_{1}$ ) as a totally real (respectively holomorphic) totally geodesic submanifold inside a complete complex hyperbolic surface $X_{2}$ (respectively $X_{1}$ ) so that: 
1. The embeddings $f_{k}: \Sigma_{k} \rightarrow X_{k}, k=1,2$ are homotopy equivalences.

2. The homotopy-inverse to $f_{k}$ is the nearest-point projection $\hat{\Pi}_{k}: X_{k} \rightarrow$ $f_{k}\left(\Sigma_{k}\right)$.

3. The numbers $\epsilon, \ell$ are chosen so that $\epsilon$-neighborhoods of distinct boundary components of $\Sigma_{k}$ are injective and disjoint.

4. Each component $f_{k}\left(\gamma_{k i}\right)$ of $f_{k}\left(\partial \Sigma_{k}\right)$ is contained in a neck $N_{k i}:=$ $\operatorname{Neck}_{\epsilon}\left(\gamma_{k i}\right)$ and necks around distinct geodesics are disjoint.

5. If two annular components

$$
A_{1 i} \subset \operatorname{Nbd}_{\epsilon}\left(\partial \Sigma_{1}\right), A_{2 i} \subset \operatorname{Nbd}_{\epsilon}\left(\partial \Sigma_{2}\right)
$$

are adjacent in the surface $\Sigma$ then there is a canonical isometry $\xi_{i}$ between the corresponding necks $N_{1 i}, N_{2 i}$.

To guarantee the properties 3-5, we need "enough room" around the boundary geodesics of $f_{k}\left(\partial \Sigma_{k}\right)$ in $X_{k}$. This is achieved by the choice of $\ell$ and $\epsilon$ as above.

Now the construction of $M$ proceeds as follows. Remove from the manifolds $X_{k}(k=1,2)$ all points in $\hat{\Pi}_{k}^{-1}\left(\partial \Sigma_{k}\right)$ that do not belong to the union of necks $N_{k i}$. The resulting complex hyperbolic manifolds with boundaries $M_{1}$ and $M_{2}$ are glued together along their necks via the isometries $\xi_{i}$. Then we prove that the resulting complex hyperbolic surface $M$ satisfies the assertions of Theorem 4.1.

The following two sections contain the details of the proof.

\subsection{Construction of the complex hyperbolic surface.}

We consider a surface $\Sigma$ as in the previous section. For each component $\Sigma_{k j}$ of the surface $\Sigma_{k}$ we have the action of $\Gamma_{k j}=\pi_{1}\left(\Sigma_{k j}\right)$ on its Nielsen region $\tilde{\Sigma}_{k j}$ in the hyperbolic plane $\mathbf{H}^{2}\left(-k^{-2}\right)$ with curvature $-k^{-2}$. Recall that $\tilde{\Sigma}_{k j}$ is the convex hull of the limit set of $\Gamma_{k j}=\pi_{1}\left(\Sigma_{k j}\right)$ and is a convex domain with totally geodesic boundary. Furthermore $\Sigma_{k j}=\tilde{\Sigma}_{k j} / \Gamma_{k j}$ is a compact geodesically convex hyperbolic surface with geodesic boundary - the convex core of a the complete hyperbolic manifold $\Sigma_{k j}=W_{k} / \Gamma_{k j}$. According to Proposition 2.6 for each $k, j$ there exists an equivariant totally geodesic embedding of the hyperbolic plane $\mathbf{H}^{2}\left(-k^{-2}\right)$ in $\mathbf{H}_{\mathbb{C}}^{2}$ which satisfies the following properties: 
1. For $k=1$ the image is the complex hyperbolic line $\mathbf{H}_{\mathbb{C}}^{1}=W_{1}$, for $k=2$ the image is a totally real hyperbolic plane $\mathbf{H}_{\mathbb{R}}^{2}=W_{2}$.

2. Each component $\beta$ of $B$ is represented by a transvection $h_{\beta}$ in $\mathbf{H}_{\mathbb{C}}^{2}$.

3. The canonical orientation on $W_{1}$ agrees with the orientation on $\Sigma_{1}$.

Thus representatives $h_{\beta}$ of all the components of $B$ are conjugate in $\mathrm{SU}(2,1)$. We let $X_{k}$ be the disjoint union of quotients of $\mathbf{H}_{\mathbb{C}}^{2} / \Gamma_{k j}$. It is clear that we have totally geodesic embeddings $f_{k}: \Sigma_{k} \hookrightarrow X_{k}$ which are homotopy equivalences. The property 2 implies that for each boundary geodesic $\beta$ of $\Sigma_{k}$ we can choose a normal frame at $f_{k}(\beta) \subset X_{k}$, see section 3.4.

These normal frames determine necks $\operatorname{Neck}_{\epsilon}(\beta) \subset X_{k}$ around the geodesics $f_{k}(\beta)$ so that necks around distinct geodesics are disjoint (see Lemma 3.8). We define submanifolds $M_{k} \subset X_{k}$ as in the previous section, and denote by $M_{k j}$ the component of $M_{k}$ containing $\Sigma_{k j}$. Each neck has a canonical fibration over an annulus, this $\mathbb{R}^{2}$-fibration extends to the fibration of

$$
M_{k}-\bigcup_{\beta \subset \partial \Sigma_{k}} N e c k_{\epsilon}(\beta)
$$

given by the nearest-point projection to $f_{k}\left(\Sigma_{k}\right)$, see section 3.2. Suppose now that $\beta_{k} \subset \partial \Sigma_{k}(k=1,2)$ are boundary geodesics identified in the surface $\Sigma$. Then there exists a unique biholomorphic isometry $\xi: \operatorname{Neck}_{\epsilon}\left(\beta_{1}\right) \rightarrow$ $\operatorname{Neck}_{\epsilon}\left(\beta_{2}\right)$ that preserves the normal frames and carries base points to base points. We let $M$ be the complex hyperbolic surface obtained via performing these gluings. Clearly the mappings $f_{k}$ can be combined to define a piecewise totally geodesic embedding

$$
f: \Sigma \hookrightarrow M
$$

which we call a spine. Similarly $\mathbb{R}^{2}$-fibrations of the components $M_{k}$ define a smooth $\mathbb{R}^{2}$-fibration $\hat{\Pi}: M \rightarrow \Sigma$. This fibration is a homotopy inverse to $f$. Moreover, $\hat{\Pi} \circ f=i d$. In the next section we shall compute the Euler number of this fibration.

Lemma 4.2. The complex hyperbolic structure on $M$ is geodesically complete.

Proof. Assume that $M$ is not complete and $\sigma:[0, T) \rightarrow M$ is a nonextendable finite geodesic ray. Each component $M_{k j}$ of $M_{k}$ is metrically complete 
by the construction and there is $\delta>0$ such that boundary components of each neck of $M$ are $\delta$-separated according to Proposition 3.1. Therefore there are infinitely many disjoint intervals $\left[t_{n}^{-}, t_{n}^{+}\right]$on $[0, T)$ such that the segment $\sigma\left(\left[t_{n}^{-}, t_{n}^{+}\right]\right)$connects different boundary components of necks in $M$. Each segment has length at least $\delta$. This contradicts the finiteness of $T$.

As a consequence of the lemma, the corresponding holonomy representations $\pi \rightarrow \mathrm{PU}(2,1)$ are discrete and faithful. The pleated surface $\tilde{f}: \tilde{\Sigma} \rightarrow \mathbf{H}_{\mathbb{C}}^{2}$ has bending angles equal to $\pi / 2$ and the bending locus consists of $2 \epsilon$-separated geodesics. According to (3.4), we can make $\epsilon$ arbitrarily large if we choose $l$ sufficiently small. Since $\mathbf{H}_{\mathbb{C}}^{2}$ has pinched negative curvature, it follows that for sufficiently large $\epsilon$, the image of $\tilde{f}$ is quasi-convex and Hausdorff close to its convex hull. Thus, the action of $G=\pi_{1}(M)$ on $\mathbf{H}_{\mathbb{C}}^{2}$ by deck transformations is convex cocompact. The action of $G$ on $\mathbf{H}_{\mathbb{C}}^{2}$ preserves a nonempty closed convex subset on which $G$ acts cocompactly.

Therefore the manifold

$$
\bar{M}=\left(\mathbf{H}_{\mathbb{C}}^{2} \cup \Omega(G)\right) / G
$$

is compact. This compactification of $M$ is consistent with the open disk bundle structure of $M$ and therefore $\bar{M}$ is diffeomorphic to the closed disk bundle over $\Sigma$ having the same Euler number as the bundle $M \rightarrow \Sigma$.

Corollary 4.3. The manifold $\Omega(G) / G$ is diffeomorphic to the total space of an $\mathbb{S}^{1}$-bundle over the surface $\Sigma$ which has the same Euler number as the $\mathbb{R}^{2}$-fibration of $M$.

\subsection{Calculation of Euler number and Toledo invariant.}

The contribution of the totally real components of $f$ to the integral

$$
\tau=\frac{1}{2 \pi} \int_{\Sigma} f^{*} \omega_{M}
$$

is zero. Thus the integral $\tau$ equals $-\chi\left(\Sigma_{1}\right)$ (see Section 2.3). This finishes the proof of the first and second assertions of Theorem 4.1.

We construct a continuous vector field $V$ along the piecewise totally geodesically embedded spine surface (4.1) as follows: Along the folding loops $f(B)$ we choose $V$ to be a parallel unit vector field orthogonal to both $f\left(\Sigma_{k}\right)$. This is possible because the holonomy along all loops $f(B)$ is trivial. Next we extend $V$ to sections of the normal bundles of the surfaces $f\left(\Sigma_{k}\right)$ which 
are smooth on $f\left(\Sigma_{k}\right)$ and have isolated zeros. By the computation in $\S 2.3$ and $\S 2.5$, the sum of the multiplicities for the zeros of $V$ equals $\chi\left(\Sigma_{1}\right) / 2$ on the complex portion $f\left(\Sigma_{1}\right)$ and $\chi\left(\Sigma_{2}\right)$ on the totally real part $f\left(\Sigma_{2}\right)$. We use the vector field $V$ to homotope $f(\Sigma)$ off itself to a nearby surface $g(\Sigma)$ which intersects $f(\Sigma)$ transversally in finitely many points. The multiplicities of the intersection points sum to the Euler number $e(M \rightarrow \Sigma)$ of the oriented $D^{2}$-fibration $M \longrightarrow \Sigma$, which equals

$$
\chi\left(\Sigma_{1}\right) / 2+\chi\left(\Sigma_{2}\right)
$$

as desired. This concludes the proof of Theorem 4.1.

Proof of Theorem 1.1. If $\tau=2 g-2,0,2-2 g$ then discrete embeddings of $\pi$ into $\mathrm{SU}(1,1)$ or $\mathrm{SO}(2,1)$ give the desired representations. Thus let $\tau$ be an even integer satisfying

$$
0<\tau<2 g-2
$$

Then

$$
t=g-1-\frac{\tau}{2}
$$

satisfies

$$
0<t<g-1 \text {. }
$$

Let $\Sigma$ denote a closed orientable surface of genus $g$. Then there exist two disjoint simple closed curves decomposing $\Sigma$ as

$$
\Sigma=\Sigma_{1} \cup \Sigma_{2}
$$

where $\Sigma_{1}$ is a surface of genus $g-t-1$ with two boundary components and $\Sigma_{2}$ is a surface of genus $t$ with two boundary components.

Thus the complex hyperbolic surface $M$ in Theorem 4.1 achieves Toledo invariant $\tau$. The negative values of $\tau$ can be obtained by conjugating these representations by an anti-holomorphic isometry of $\mathbf{H}_{\mathbb{C}}^{2}$. Finally,

$$
e(M \rightarrow \Sigma)-\chi(\Sigma)=|\tau| / 2
$$

follows from direct computation.

Using degree one maps between hyperbolic surfaces and complex Fuchsian representations one can easily construct nonfaithful discrete representations of $\pi_{1}\left(\Sigma_{g}\right)$ realizing all even integer values of Toledo's invariant. These representations will have images in $\mathrm{SU}(1,1)$. 


\section{Examples with nonintegral Toledo invariant.}

In this section we construct representations of the fundamental group $\pi=$ $\pi_{1}(\Sigma)$ that realize all possible fractional values of the invariant $\tau(\rho)$. We start with the case of surfaces of genus 2 .

Proposition 5.1. Suppose that $\Sigma$ is a closed oriented surface of genus 2. Then there are representations $\rho_{j}: \pi=\pi_{1}(\Sigma) \rightarrow \mathrm{PU}(2,1)$ so that $\tau\left(\rho_{j}\right)=$ $2 j / 3(j=1,2)$.

Proof. Consider a family of regular quadrilaterals

$$
Q_{\delta}, \quad 0<\delta<\pi / 2
$$

in $\mathbf{H}_{\mathbb{C}}^{1} \subset \mathbf{H}_{\mathbb{C}}^{2}$ such that all angles of $Q_{\delta}$ equal $\delta$. Let $A_{\delta}, B_{\delta}$ be transvections in $\mathrm{PU}(2,1)$ pairing the opposite sides of $Q_{\delta}$. The commutator $C_{\delta}=\left[A_{\delta}, B_{\delta}\right]$ is an elliptic element fixing a corner of $Q_{\delta}$. It rotates by the angle $4 \delta$ inside $\mathbf{H}_{\mathbb{C}}^{1}$ and by the angle $\pi+2 \delta$ in the normal direction. Indeed, according to Corollary 2.2 the speed of rotation in the normal direction is half of the speed of rotation in $\mathbf{H}_{\mathbb{C}}^{1}$, thus it is either $\pi+2 \delta$ or $2 \delta$. Since

$$
\lim _{\delta \rightarrow \pi / 2} C_{\delta}=i d,
$$

the rotation angle equals $\pi+2 \delta$. We now restrict to the special value $\delta=\pi / 6$, then the angles of rotation in $\mathbf{H}_{\mathbb{C}}^{1}$ and in the normal direction are $2 \pi / 3$ and

$$
2 \delta+\pi=4 \pi / 3,
$$

respectively. The elliptic element $C_{\delta}$ has order 3 . We similarly define hyperbolic elements $A_{\delta}^{\prime}, B_{\delta}^{\prime} \in \mathrm{SO}(2,1)$ whose commutator $C_{\delta}^{\prime}$ has order 3 . The eigenvalues of the derivative of $C_{\delta}^{\prime}$ at its fixed point are $e^{2 \pi / 3 i}$ and $e^{-2 \pi / 3 i}$. We consider the corresponding (discrete and non-faithful) representation

$$
\phi: F_{2}=\langle\alpha, \beta\rangle \rightarrow \mathrm{SU}(1,1) \subset \mathrm{PU}(2,1)
$$

of the free group on two generators given by $\phi(\alpha)=A_{\delta}$ and $\phi(\beta)=B_{\delta}$. The normalizer of the order 3 element $C_{\delta}$ in $\mathrm{PU}(2,1)$ contains an involution $\theta$ with which it anticommutes: $\theta C_{\delta} \theta^{-1}=C_{\delta}^{-1}$. The involution $\theta$ interchanges the eigenspaces of the derivative of $C_{\delta}$ at its fixed point, i.e. the direction tangent to $\mathbf{H}_{\mathbb{C}}^{1}$ and the normal direction. Similarly we have the representation

$$
\phi^{\prime}: F_{2}=\langle\alpha, \beta\rangle \rightarrow \mathrm{SO}(2,1) \subset \mathrm{PU}(2,1)
$$


given by $\phi^{\prime}(\alpha)=A_{\delta}^{\prime}$ and $\phi^{\prime}(\beta)=B_{\delta}^{\prime}$, which preserves a totally real geodesic plane.

We use the representation $\phi$ as a building block to construct a representation

$$
\rho_{2}: \pi=\pi_{1}(\Sigma)=\left\langle\alpha_{1}, \beta_{1}, \alpha_{2}, \beta_{2} \mid\left[\alpha_{1}, \beta_{1}\right]\left[\alpha_{2}, \beta_{2}\right]=1\right\rangle \rightarrow \mathrm{PU}(2,1)
$$

by sending

$$
\begin{aligned}
& \alpha_{1} \longmapsto \phi(\alpha), \\
& \beta_{1} \longmapsto \phi(\beta), \\
& \alpha_{2} \longmapsto \theta \phi(\alpha) \theta^{-1} \\
& \beta_{2} \longmapsto \theta \phi(\beta) \theta^{-1} .
\end{aligned}
$$

Now we compute Toledo's invariant for the representation $\rho_{2}$. Identifying the sides of $Q_{\delta}$ via the transformations $A_{\delta}, B_{\delta}$ gives a hyperbolic cone structure on the 2 -torus $T$ with a single cone point $k$ with total angle $4 \delta=2 \pi / 3$, see [13]. (This cone structure is actually an orbifold structure.) The punctured surface $S=T-\{k\}$ has an incomplete hyperbolic structure whose developing map $d: \tilde{S} \rightarrow \mathbf{H}_{\mathbb{C}}^{1}$ maps a fundamental domain in $\tilde{S}$ to $Q_{\delta}$. The homomorphism $\phi$ is the holonomy of this hyperbolic cone structure. We decompose $\Sigma$ along a simple loop $c$ into two punctured tori $\Sigma_{j}$ so that $\pi_{1}\left(\Sigma_{j}\right)$ is identified with the subgroup in $\pi_{1}(\Sigma)$ generated by $\alpha_{j}, \beta_{j}$. We choose orientation preserving diffeomorphisms $h_{j}: \Sigma_{j} \rightarrow S$ which map a neighborhood of $c$ to a neighborhood of $k$. These diffeomorphisms give rise to equivariant developing maps

$$
f_{1}=d \circ \tilde{h}_{1}: \tilde{\Sigma}_{1} \rightarrow W_{1}, \quad f_{2}=\theta \circ d \circ \tilde{h}_{2}: \tilde{\Sigma}_{2} \rightarrow \theta\left(W_{1}\right) .
$$

The complex lines $W_{1}$ and $\theta\left(W_{1}\right)$ are orthogonal and intersect in the fixed point of the elliptic isometry

$$
\rho_{2}\left(\left[\alpha_{1}, \beta_{1}\right]\right)=\rho_{2}\left(\left[\alpha_{2}, \beta_{2}\right]\right)^{-1} .
$$

The equivariant maps $f_{1}, f_{2}$ extend uniquely to a $\rho_{2}$-equivariant map $\tilde{f}$ : $\tilde{\Sigma} \rightarrow \mathbf{H}_{\mathbb{C}}^{2}$. There is a fundamental domain $\Omega$ for the action of $\pi$ on $\tilde{\Sigma}$ which maps onto the union $Q_{\delta} \cup \theta\left(Q_{\delta}\right)$ preserving orientation. Thus

$$
\tau\left(\rho_{2}\right)=\frac{1}{2 \pi} \int_{\Omega} \tilde{f}^{*} \omega=\frac{1}{\pi} \cdot \operatorname{area}(S)=\frac{1}{\pi}(2 \pi-4 \delta)=\frac{4}{3} .
$$


Now we find a representation $\rho_{1}$ with $\tau\left(\rho_{1}\right)=\frac{2}{3}$ by a similar construction. We leave

$$
\left.\rho_{1}\right|_{\pi_{1}\left(\Sigma_{1}\right)}=\left.\rho_{2}\right|_{\pi_{1}\left(\Sigma_{1}\right)}
$$

as above and replace $\left.\rho_{2}\right|_{\pi_{1}\left(\Sigma_{2}\right)}$ by setting $\rho_{1}$ on $\pi_{1}\left(\Sigma_{2}\right)$ by

$$
\begin{aligned}
& \rho_{1}: \alpha_{2} \mapsto g \phi^{\prime}(\alpha) g^{-1} \\
& \rho_{1}: \beta_{2} \mapsto g \phi^{\prime}(\beta) g^{-1} .
\end{aligned}
$$

where $\phi^{\prime}$ is defined as in (5.1) and $g$ is defined as follows. Since $\phi([\alpha, \beta])$ and $\phi^{\prime}([\alpha, \beta])^{-1}$ have the same eigenvalues, there exists an isometry $g$ of $\mathbf{H}_{\mathbb{C}}^{2}$ which conjugates $\phi^{\prime}([\alpha, \beta])$ to $\phi([\alpha, \beta])^{-1}$. By $(5.2)$,

$$
\rho_{1}\left(\left[\alpha_{1}, \beta_{1}\right]\right)=\rho_{1}\left(\left[\alpha_{2}, \beta_{2}\right]\right)^{-1},
$$

so $\rho_{1}$ is a representation $\rho_{1}: \pi \rightarrow \mathrm{PU}(2,1)$. Now

$$
\tau\left(\rho_{1}\right)=\frac{2}{3}
$$

because the totally real part $\left.\rho_{1}\right|_{\pi_{1}\left(\Sigma_{2}\right)}$ does not contribute to the Toledo invariant. This completes the proof of the proposition.

Note that no representation $\rho: \pi \rightarrow \mathrm{PU}(2,1)$ with non-integer Toledo invariant preserves a complex geodesic. The stabilizer of a complex geodesic in $\mathrm{PU}(2,1)$ is conjugate to $\mathrm{SU}(1,1)$ and therefore lifts canonically to $\mathrm{SU}(2,1)$.

Proposition 5.2. The image of $\rho_{2}$ is a discrete subgroup of $\mathrm{PU}(2,1)$.

Proof. We shall embed the image of $\rho_{2}$ in an arithmetic lattice $\Gamma \subset \mathrm{PU}(2,1)$. Consider the Hermitian form

$$
H(z)=z_{1} \bar{z}_{1}+z_{2} \bar{z}_{2}-\sqrt{3} z_{3} \bar{z}_{3}
$$

on $\mathbb{C}^{3}$ and let $G$ be the group of all linear transformations $\mathbb{C}^{3} \longrightarrow \mathbb{C}^{3}$ unitary with respect to $H$.

The group $G$ is the group of $\mathbb{R}$-points in an $\mathbb{R}$-algebraic group. Let $G_{\mathfrak{O}}$ be the subgroup of $G$ with entries from the subring $\mathfrak{O}=\mathbb{Z}[\exp (\pi i / 12)]$ where $i=\sqrt{-1}$.

Let $\sigma$ be the embedding $\mathfrak{O} \hookrightarrow \mathbb{C}$ defined by the Galois conjugation

$$
\exp (\pi i / 12)) \longmapsto \exp (\pi 5 i / 12)) .
$$


Note that $\sigma(i)=i$ and $\sigma(\sqrt{3})=-\sqrt{3}$. Then $\sigma$ transforms $G_{\mathfrak{D}}$ into the unitary group $G_{\mathfrak{O}}^{\sigma}$ of the definite Hermitian form

$$
H^{\sigma}(z)=z_{1} \bar{z}_{1}+z_{2} \bar{z}_{2}+\sqrt{3} z_{3} \bar{z}_{3} .
$$

Let $G^{\sigma}$ denote the unitary group fo $H^{\sigma}$ with entries from $\mathbb{C}$.

The graph $(I, \sigma)$ of $\sigma$ embeds $\mathfrak{O}$ as a lattice in $\mathbb{C} \times \mathbb{C}$ and realizes $G_{\mathfrak{D}}$ as an arithmetic lattice in the product $G \times G^{\sigma}$. Since $G^{\sigma}$ is compact, the projection

$$
G \times G^{\sigma} \longrightarrow G
$$

is proper, hence $G_{\mathfrak{O}}$ is a lattice in $G$.

To realize $\rho_{2}$ in $G$, we first note that the involution $\theta$ is contained in $G_{\mathfrak{D}}$. Next we will show that a $(2,4,12)$-triangle group $\Gamma_{2,4,12}$ could be embedded in $\mathrm{PU}(1,1)$ so that the image is commensurable with $G_{\mathfrak{O}} \cap \mathrm{PU}(1,1)$. (Figure 4 depicts the tessellation of $\mathbf{H}_{\mathbb{C}}^{1}$ corresponding to the reflection group which contains $\Gamma_{2,4,12}$ as a subgroup of index 2). Finally, the group $\phi\left(\pi_{1}\left(\Sigma_{1}\right)\right)$ is contained in $\Gamma_{2,4,12}$ as a finite index subgroup.

Here is the detailed construction. Consider a triangle $\Delta \subset \mathbf{H}_{\mathbb{C}}^{1}$ with a vertex $p$ at the origin, with angle $\pi / 12$, another vertex $q$ with angle $\pi / 4$ and a third vertex $r$ with right angle. The products of reflections in the lines $\overleftrightarrow{p q}, \overleftrightarrow{q r}, \overleftrightarrow{r p}$, are rotations $P, Q, R$ in the points $p, q, r$ respectively. These isometries of $\mathbf{H}_{\mathbb{C}}^{1}$ satisfy

$$
P^{12}=Q^{4}=R^{2}=P Q R=I
$$

and any triple $(P, Q, R)$ satisfying these relations, where $P$ (respectively $Q, R)$ represents elliptic rotation through angle $\pi / 6$ (respectively $\pi / 2, \pi$ ) corresponds to a triangle $\Delta$. Explicitly, such a group is represented by the matrices

$$
P=\left[\begin{array}{cc}
\exp (-\pi i / 12) & 0 \\
0 & \exp (\pi i / 12)
\end{array}\right]
$$

and

$$
Q=\left[\begin{array}{lc}
1+i(2+\sqrt{3}) & -i(1+\sqrt{3}) \\
i \sqrt{3}(1+\sqrt{3}) & 1-i(2+\sqrt{3})
\end{array}\right]
$$

and

$$
R=\left[\begin{array}{cc}
i(1+\sqrt{3}) & \frac{1}{2}(1-(2+\sqrt{3})) \\
\frac{\sqrt{3}}{2}(1+i(2+\sqrt{3})) & -i(1+\sqrt{3})
\end{array}\right]
$$


where

$$
\begin{aligned}
\omega & =e^{2 \pi i / 3}=(-1+i \sqrt{3}) / 2 \\
\exp (3 \pi i / 4) & =\frac{1-i}{\sqrt{2}} \\
\exp (\pi i / 12) & =-\exp (3 \pi i / 4) \bar{\omega}=\frac{(1+\sqrt{3})+i(-1+\sqrt{3})}{2 \sqrt{2}} .
\end{aligned}
$$

Since the projection from $2 \times 2$ matrices to $\mathrm{PU}(1,1)$ is not faithful, an isometry of even order $2 m$ lifts to a matrix whose $2 m$-th power is $-I$ (not $I)$. One can easily check that the above matrices satisfy relations

$$
P^{12}=Q^{4}=R^{2}=P Q R=-I .
$$

Thus $\Gamma_{2,4,12} \subset G_{\mathcal{O}_{\tilde{j}}}$ We now realize $\phi\left(\pi_{1}\left(\Sigma_{1}\right)\right)$ in $\Gamma_{2,4,12}$. We find the generators $\tilde{\alpha}=\phi(\alpha), \tilde{\beta}=\phi(\beta)$ for a finite index subgroup of $\Gamma_{2,4,12}$ as follows. Begin with the free product

$$
\mathbb{Z} / 4 \star \mathbb{Z} / 2=\langle X\rangle *\langle Y\rangle=\left\langle X, Y \mid X^{2}=Y^{4}=I\right\rangle
$$

and consider the homomorphism

$$
\begin{aligned}
\eta: \mathbb{Z} / 2 \star \mathbb{Z} / 4 & \longrightarrow \mathbb{Z} / 4 \\
X & \longmapsto 2 \bmod 4 \\
Y & \longmapsto 1 \bmod 4 .
\end{aligned}
$$

Then $Y X Y$ and $X Y^{2}$ generate the kernel of $\eta$. To see this, replace

$$
\left\langle X, Y \mid X^{2}=Y^{4}=I\right\rangle
$$

by the equivalent presentation

$$
\left\langle Y, A, B \mid Y^{4}=I, Y A Y^{-1}=B^{-1}, Y B Y^{-1}=A\right\rangle,
$$

where $X$ corresponds to $B Y^{2}$ and $A$ (respectively $B$ ) corresponds to $Y X Y$ (respectively $X Y^{2}$ ). In the new presentation $\eta$ maps $Y$ to a generator of $\mathbb{Z} / 4$ and annihilates $A, B$, so $A, B$ generate $\operatorname{ker}(\eta)$. Their commutator is easily computed to be:

$$
\left[Y X Y, X Y^{2}\right]=(Y X)^{4} .
$$

Now apply this to the homomorphism

$$
\mathbb{Z} / 2 \star \mathbb{Z} / 4 \longrightarrow \Gamma_{2,4,12}
$$


which maps $X$ to $Q, Y$ to $R$ and $X Y$ to $P$. The homomorphism $\eta$ projects to an epimorphism

$$
\tilde{\eta}: \Gamma_{2,4,12} \longrightarrow \mathbb{Z} / 4
$$

and we obtain elements $\tilde{\alpha}, \tilde{\beta} \in \operatorname{ker}(\tilde{\eta})$ as

$$
\tilde{\alpha}=Q R Q, \tilde{\beta}=R Q^{2} .
$$

Since $\chi\left(\Gamma_{2,4,12}\right)=-1 / 4$, the index four subgroup $\operatorname{ker}(\tilde{\eta})$ satisfies $\chi(\operatorname{ker}(\tilde{\eta}))=$ -1 and thus is free of rank two. It follows that $\tilde{\alpha}, \tilde{\beta}$ generate $\operatorname{ker}(\tilde{\eta}))$.

Now define the homomorphism $\phi$ on the free group $F_{2}=\langle\alpha, \beta\rangle$ by

$$
\phi(\alpha)=\tilde{\alpha}=Q R Q=\left[\begin{array}{cc}
(1+\sqrt{3}) \bar{\omega} & -\bar{\omega}+i \\
-\sqrt{3}(\omega+i) & (1+\sqrt{3}) \omega
\end{array}\right]
$$

and

$$
\phi(\alpha)=\tilde{\alpha}=Q R Q=\left[\begin{array}{cc}
(1+\sqrt{3}) \omega & -\omega-i \\
-\sqrt{3}(\bar{\omega}-i) & (1+\sqrt{3}) \bar{\omega}
\end{array}\right]
$$

Then the commutator

$$
\phi([\alpha, \beta])=P^{-4}=\left[\begin{array}{cc}
\omega & 0 \\
0 & \bar{\omega}
\end{array}\right]
$$

is the desired elliptic element of order 3 .

Applying the construction of the proof of Proposition 5.1, we see that $\theta \in G_{\mathfrak{O}}$ and thus the image of $\rho_{2}$ lies in the arithmetic lattice $G_{\mathfrak{O}}$ and is therefore discrete.

Proof of Theorem 1.1. We modify the construction in the proof of Proposition 5.1 by amalgamating representations coming from holonomy representations of hyperbolic cone structures on surfaces of higher genus. Let $\Sigma$ be a closed surface of genus $g \geq 2$ which is split along a separating simple loop $c$ into two subsurfaces $\Sigma_{1}$ and $\Sigma_{2}$ of genera $g_{1}=g-1$ and $g_{2}=1$, respectively. We pick singular hyperbolic surfaces $T_{j}(j=1,2)$ of genus $g_{j}$ with one cone point $k_{j}$ of angle $\sigma$; such surfaces can be constructed by suitably identifying the sides of a regular hyperbolic $4 g_{j}$-gon with angles $\sigma / 4 g_{j}$. Since $\pi_{1}\left(T_{j}-\left\{k_{j}\right\}\right)$ is a free group, the holonomy representation

$$
\operatorname{hol}_{j}: \pi_{1}\left(T_{j}-\left\{k_{j}\right\}\right) \rightarrow \mathrm{PU}(1,1)
$$




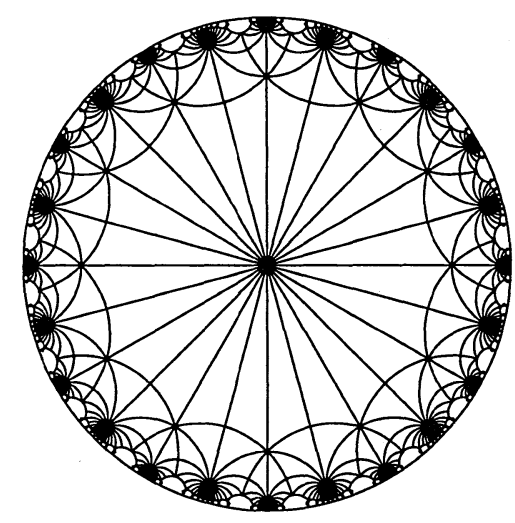

Figure 4: The $(2,4,12)$ Schwarz triangle group

for the singular hyperbolic structure on $T_{j}$ lifts to a representation

$$
\widetilde{\operatorname{hol}}_{j}: \pi_{1}\left(T_{j}-\left\{k_{j}\right\}\right) \rightarrow \mathrm{SU}(1,1) \subset \mathrm{PU}(2,1)
$$

Let $W$ be the complex geodesic preserved by $\mathrm{SU}(1,1)$. An element $\gamma$ representing a small simple loop around $k_{j}$ is carried by hol ${ }_{j}$ to a rotation in $W$ by angle $\sigma$ around a point $o \in W$. The lifted holonomy $\widetilde{\operatorname{hol}}_{j}(\gamma)$ acts as a rotation by angle $\pi+\sigma / 2$ in the normal direction to $W$ at the point $o$. For the genus one case this was already discussed in the proof of Proposition 5.1. The angle of rotation in the normal direction is either $\sigma / 2$ or $\pi+\sigma / 2$. To exclude the first possibility, we note that the hyperbolic cone structure of $T_{j}$ can be continuously deformed so that $\sigma$ increases to $2 \pi$. The limit is a nonsingular hyperbolic structure whose holonomy can be lifted to $\mathrm{SU}(1,1)$, see $[11,4]$. Hence the assertion follows by continuity.

Now we restrict $\sigma$ to the special value $2 \pi / 3$ and proceed as in the proof of Proposition 5.1. By combining $\widetilde{\mathrm{hol}}_{1}$ and $\widetilde{\mathrm{hol}}_{2}$ we construct a representation with Toledo invariant $-\chi(\Sigma)-2 / 3$. By combining $\widetilde{\text { hol}}_{1}$ with the appropriate conjugate of the representation $\phi^{\prime}$ we get a representation with Toledo invariant $-\chi(\Sigma)-4 / 3$. Thus we can realize the extremal positive non-integer values

$$
-\chi(\Sigma)-2 / 3, \quad-\chi(\Sigma)-4 / 3
$$

for Toledo's invariant. To realize the values $2 k-2 / 3$ and $2 k-4 / 3$ for $k=1, \ldots, g-2$ choose a degree-one map of $\Sigma$ onto a surface $\Sigma^{\prime}$ of genus $k+1$. Take representations $\rho^{\prime}: \Sigma^{\prime} \rightarrow \mathrm{PU}(2,1)$ with Toledo's invariant $2 k-2 / 3$ and $2 k-4 / 3$ as constructed above and compose them with the 
induced map $\pi_{1}(\Sigma) \rightarrow \pi_{1}\left(\Sigma^{\prime}\right)$ of fundamental groups. This yields all positive fractional values for Toledo's invariant. The negative values are obtained by conjugating the above representations with an antiholomorphic isometry of $\mathbf{H}_{\mathbb{C}}^{2}$.

\section{References.}

[1] A.F. Beardon, The Geometry of Discrete Groups, Graduate Texts in Mathematics 91, Springer-Verlag, New York - Heidelberg - Berlin (1983).

[2] D. Burns and S. Shnider, Spherical hypersurfaces in complex manifolds, Inv. Math., 33 (1976), 223-246.

[3] J. Dupont, Simplicial de Rham cohomology and characteristic classes of flat bundles, Topology, 15 (1976), 233-245.

[4] W.M. Goldman, Topological components of spaces of representations, Inv. Math., 93 (1988), 557-607.

[5] Complex Hyperbolic Geometry, Oxford Mathematical Monographs, Oxford University Press (1999).

[6] and M. Kapovich, Complex hyperbolic surfaces homotopy equivalent to a Riemann surface, MSRI Preprint, 1992.

[7] P.A. Griffiths and J. Harris, Principles of Algebraic Geometry, John Wiley \& Sons, New York (1978).

[8] M. Kapovich and B. Leeb, (in preparation).

[9] H. Karcher, Riemannian comparison constructions, in: S.S. Chern ed., Global Differential Geometry, MAA studies in Mathematics, vol. 27 (1989), 170- 222.

[10] S. Kobayashi and K. Nomizu, Foundations of Differential Geometry I, Interscience Tracts in Pure and Applied Mathematics, 15, John Wiley \& Sons, New York (1969).

[11] H. Petersson, Zur analytischen Theorie der Grenzkreisgruppen III, Math. Ann. 115 (1938), 518-572.

[12] N. Steenrod, The Topology of Fibre Bundles, Princeton University Press, 1951.

[13] W. Thurston, Shapes of polyhedra and triangulations of the sphere, 511-549, Geometry and Topology Monographs I: The Epstein Birthday Schrift (I. Rivin, C. Rourke, C. Series eds.), (1998) International Press. 
[14] D. Toledo, Harmonic maps from surfaces to certain Kähler manifolds, Math. Scand., 45 (1979), 13- 26.

[15] - Representations of surface groups on complex hyperbolic space, J. Diff. Geom., 29 (1989), 125-133.

[16] E. Xia, The moduli of flat $U(p, q)$ structures over Riemann surfaces, (submitted).

RECEIVED OCTOBER 26, 1998.

UNIVERSITY OF MARYLAND

College Park, MD 20742

USA

UNIVERSITY OF UTAH,

SALt LAKE City, Utah 84112

USA

AND

EBERHARD-KaRLS-UNIVERSITÄT TÜBINGEN, D-72076 TÜBINGEN,

GERMANY

E-mail addresses: wmg@math.umd.edu kapovich@math.utah.edu

leeb@moebius . mathematik . uni-tuebingen. de 